\title{
Terrains de jeu et stèles dans la région maya de Río Bec : rejet ou inutilité ?
}

Ballcourts and stelae in the Río Bec region. Rejection or uselessness?

Juegos de pelota y estelas en la región Río Bec: ¿rechazo o inutilidad?

\section{Eric Taladoire}

\section{(2) OpenEdition}

Journals

Édition électronique

URL : https://journals.openedition.org/jsa/14062

DOI : 10.4000/jsa. 14062

ISSN : 1957-7842

Éditeur

Société des américanistes

Édition imprimée

Date de publication : 31 décembre 2014

Pagination : 145-177

ISSN : 0037-9174

Référence électronique

Eric Taladoire, "Terrains de jeu et stèles dans la région maya de Río Bec : rejet ou inutilité ? ", Journal de la Société des américanistes [En ligne], 100-2 | 2014, mis en ligne le 01 janvier 2016, consulté le 05 septembre 2022. URL : http://journals.openedition.org/jsa/14062 ; DOI : https://doi.org/10.4000/ jsa. 14062 


\title{
TERRAINS DE JEU ET STÈLES \\ DANS LA RÉGION MAYA DE RÍO BEC : REJET OU INUTILITÉ ?
}

\author{
Éric TALADOIRE *
}

La région Río Bec est connue pour son style architectural et son organisation spatiale en groupes d'édifices de dimensions assez comparables. Elle se caractérise aussi par l'absence relative de monuments à inscriptions et de terrains de jeu de balle. Ce travail cherche à vérifier si cette rareté apparente repose sur des faits bien établis, et à expliquer certaines des raisons de ce phénomène, en fonction de l'organisation socio-politique régionale. [Mots-clés: Río Bec, jeu de balle, stèles, inscriptions, organisation socio-politique.]

Ballcourts and stelae in the Río Bec region. Rejection or uselessness? The Río Bec region is renowned for its specific architectural style and its spatial organization in more or less equivalent groups of buildings. It is also characterized by its relative absence of inscribed monuments and ballcourts. In this contribution, we try to establish if this apparent scarcity reflects a real absence, and to interpret consequently the reasons of this phenomenon, as related to the regional sociopolitical organization. [Key words: Río Bec, ballcourts, stelae, inscriptions, sociopolitical organization.]

Juegos de pelota y estelas en la región Río Bec: ¿rechazo o inutilidad? La región Río Bec es famosa por su estilo arquitectónico específico y su organización espacial en grupos de edificios de dimensiones más o menos equivalentes. Pero se caracteriza también por la escasez de monumentos con inscripciones y de canchas de juego de pelota. Este trabajo busca verificar si esta escasez aparente queda confirmada, y entender las razones de este fenómeno en el marco de la estructura socio-política regional. [Palabras claves: Río Bec, juegos de pelota, estelas, inscripciones organización socio-política.]

La rareté des terrains de jeu de balle dans les sites de la zone Río Bec (Figure 1) constituerait un des traits caractéristiques de cette région, parallèlement à la quasi-absence de stèles ou de monuments sculptés porteurs d'inscriptions. Ce point a été discuté à diverses reprises dans les rapports du projet Río Bec

* UMR 8096, Archéologie des Amériques, université Paris 1 Panthéon-Sorbonne [E.Taladoire@ univ-paris1.fr].

Journal de la Société des américanistes, 2014, 100-2, pp. 145-177. @ Société des américanistes. 


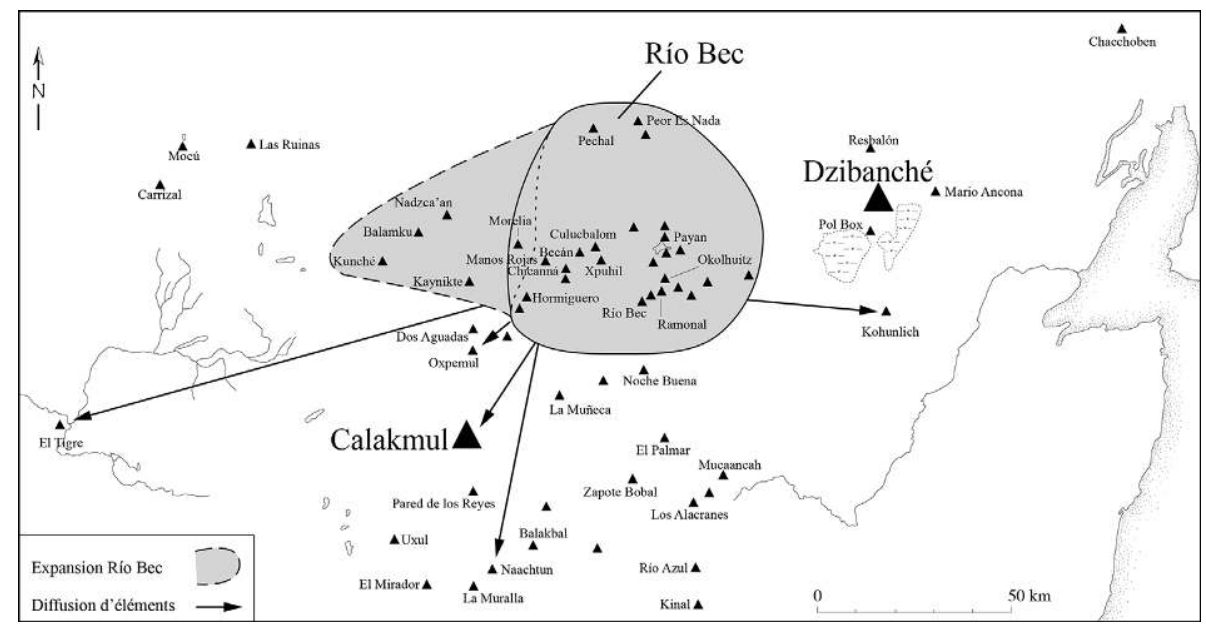

Fig. 1 - Carte de localisation de l'aire Río Bec et de sa zone d'expansion maximale (d'après Nondédéo et al. 2010).

(Michelet et al. 2005a). Il ne sera pas ici question des critères stylistiques de l'architecture résidentielle qui ont donné lieu à de nombreuses études $(e . g$. Andrews 1999 ; Bueno 1999; Gendrop 1983), et dont les spécificités ont fait l'objet de recherches ciblées au cours du Projet (Arnauld et al. 2013 ; Fowler et Arnauld 2013 ; Nondédéo et al. 2013 ; Nondédéo et Patrois 2007 ; Taladoire et al. 2013). Seules des absences ou des critères considérés, par comparaison avec les autres régions de l'aire maya, comme "négatifs » feront ici l'objet de notre attention.

Plusieurs questions se posent donc pour les rares cas connus de terrains et de stèles de la zone Río Bec. La première est celle de leur identification. On peut la supposer résolue, au moins pour ce qui est des terrains des Groupes II et V de Río Bec (ci-dessous), et du terrain de Becán, même si des doutes subsistent pour les autres. La seconde est celle de leur datation. Sont-ils antérieurs à l'apogée du phénomène régional ? Si tel fut le cas, ont-ils été utilisés malgré tout durant le Classique récent, ou étaient-ils abandonnés ? Une troisième question découle des précédentes. Si la pratique du jeu demeure limitée, ou diminue à l'apogée du phénomène Río Bec, comme cela semble être le cas, quelle en est la cause ? Même si certains terrains de Río Bec restent utilisés, leur rareté soulève une question corollaire : comment comprendre que ce trait culturel ait connu un effacement relatif ? Doit-on y voir un rejet ou une inutilité dans une région dont la particularité est l'absence de véritable «centre » à la population concentrée et aux monuments à fonctions publiques ? Seule une étude régionale permet d'avancer une réponse. 


\section{LES QUESTIONS DE QUANTIFICATION}

Commençons par les stèles, parce qu'elles permettent de poser immédiatement le cadre régional et la quantité de sites archéologiques à inventorier (Figure 2).

En 1999, selon Bueno (Nondédéo 2003, p. 82), le total des stèles et des monuments sculptés s'élevait à 29 pour cinq sites de l'ensemble de la région, soit Becán (9), Río Bec (Groupes II : 5 et V : 6), Pasión del Cristo (3), Pechal (5) et El Coyote (1). Les recherches récentes ont accru cette courte liste de deux monuments (10 et 11) à Becán (Campaña 2005), cinq stèles (dont une lisse) sur le site de Kajtún et deux autres dans le groupe proche de Dzibil (Nondédéo et Lacadena 2004). Les sites de Pueblo Viejo B, San Lorenzo (Thompson 1953 ; Mayer 2003) et Xpuhil VIII comptent chacun un monument, et celui de Manos Rojas 3, trois occurrences supplémentaires (Nondédéo et Patrois 2007, note 3). Une dalle peinte de Becán a été publiée par Mathews (1983), une autre enregistrée à Chicanná (Mayer 1988). Les banquettes de la structure 6N-2 (Arnauld et Lacadena 2004) complètent ce total, même s'il s'agit d'un support différent. Sur plus de 156 sites ou groupes majeurs de style Río Bec identifiés (Nondédéo et Patrois 2007), le total de ceux qui comptent des stèles s'établit désormais à onze avec 44 monuments ou stèles ${ }^{1}$. On observe, par ailleurs, une concentration dans deux secteurs, Becán et la zone de Río Bec proprement dite (Groupes II, V, Kajtún et Dzibil) qui comptent à eux seuls près des deux tiers du corpus (29 exemples). D'autre part, 9 des 44 stèles sont lisses, 13 autres le sont probablement, soit $50 \%$ du total. Le placement chronologique et la localisation doivent enfin être pris en compte pour l'analyse. En effet, le Groupe II de Río Bec a, par exemple, connu une occupation du Classique ancien et au moins l'une des stèles (la 5) se rattache à cette occupation, ce qui exclut son appartenance au style régional, puisqu'elle ne correspond pas au développement du phénomène Río Bec (Nondédéo 2003). Pechal, enfin, se situe aux marges septentrionales de l'aire Río Bec, ce qui soulève la question de l'appartenance des cinq stèles qui s'y trouvent à la tradition régionale.

Pour les terrains de jeu de balle, Nondédéo (2003, p. 83, note 12) fait état de l'existence de quatre exemples, un à Becán (avec deux étapes de construction), un à Peor es Nada et deux à Río Bec (Groupes II et V) (Ruppert et Denison 1943 ; Taladoire 1981). Il ajoute que, selon Andrews (non daté), il en existerait un cinquième dont la localisation n'est pas précisée. Benavides (1996), dans le cadre du projet Manzana, en a peut-être identifié un autre sur le site de Puerto Rico, qui pourrait être celui signalé par Andrews, qui a visité ce site. Le nombre de terrains identifiés dans la région de Río Bec est donc extrêmement réduit : 5, 6 au plus (Figure 2). D'autres terrains existent dans des sites où s'est manifestée l'influence Río Bec, à Kohunlich, Nadzca'an ou Balamkú, mais leur présence daterait plutôt 
d'occupations antérieures. On doit noter que les Groupes II et V différent des autres groupes de Río Bec par la présence de terrains, de stèles et, pour le premier, d'une organisation spatiale de type Petén, avec un complexe de rituel public caractéristique. Les données chronologiques disponibles suggèrent, au moins pour ce même groupe, une occupation antérieure à l'apogée du phénomène Río Bec.

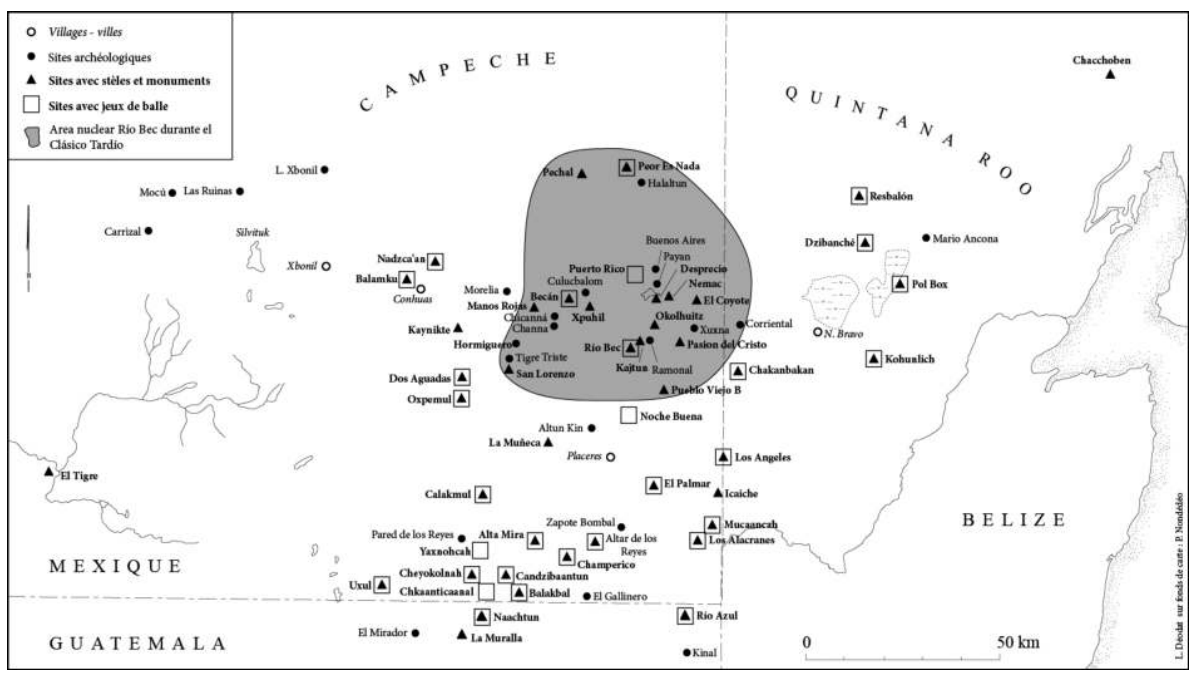

FIG. 2 - Carte de répartition régionale des terrains de jeu de balle et des monuments sculptés.

\section{L'ABSENCE RELATIVE DE TERRAINS DE JEU DE BALLE}

Nondédéo (2003) n'était pourtant pas convaincu du caractère discriminant de l'absence de cet élément culturel. Il soulignait que, dans le Sud-Est du Campeche et les zones limitrophes, le nombre de terrains connus demeurait faible : seuls les sites de Calakmul, Uxul, Balakbal, El Palmar, Dos Aguadas et Mucaancah en comptent un, tandis que des sites majeurs comme La Muñeca, Naachtun ${ }^{2}$ ou Oxpemul en seraient dépourvus. Les travaux récents ont toutefois confirmé leur existence à Oxpemul (Folan et al. 2005 ; Špracj et al. 2008) et Naachtun (ReeseTaylor et al. 2005). Nondédéo ne fait pas état, en revanche, de l'existence des terrains de Balamkú et Nadzca'an (2), ni de ceux de plusieurs sites voisins du Quintana Roo (Dzibanché, Kohunlich, Chakanbakan) ou du nord du Petén (Río Azul), localisés, il est vrai, hors de sa zone de recherche.

Le même constat de rareté des terrains dans les Basses Terres septentrionales a été formulé par d'autres auteurs (Greene Robertson et al. 1991) et repris plus récemment pour le Quintana Roo (Shaw et Mathews 2005, passim). 
Sans contester l'hypothèse d'une densité moindre de terrains pour les Basses Terres septentrionales par comparaison avec les Basses Terres centrales ${ }^{3}$, la disproportion est toutefois moins patente qu'on ne le suppose et tient plutôt au manque de recherches (Taladoire 1993) ${ }^{4}$. Cette explication serait d'autant plus valable pour le Quintana Roo que les fouilles archéologiques restent insuffisantes. Les travaux en cours ont d'ailleurs substantiellement accru le corpus, avec de nouveaux exemples découverts à Lagartera et Yo'okop (Shaw et Mathews 2005 ; Normack 2009), dans la région de Cochuah ou à Pol Box (Esparza Olguín et Pérez Gutiérrez 2009) ${ }^{5}$. Dans ce contexte, l'absence effective de terrains identifiés dans la région de Río Bec est d'autant plus manifeste, puisque la multiplication récente des travaux n'a pas accru le total déjà connu ${ }^{6}$.

Les recherches récentes, à Calakmul (Carrasco 1996), Balamkú (Michelet et al. 1998), Nadzca'an (Pescador 1998), Kohunlich, Pol Box (Esparza Olguín et Pérez Gutiérrez 2009) ou Dzibanché (Nalda et al. 1994) et la reconnaissance menée dans la zone voisine du Sud-Est du Campeche (Špracj et al. 2008) montrent en revanche que le nombre de terrains est plus important dans les sites voisins, que ce soit à la fin du Classique ancien et au Classique récent. Leur nombre s'élève actuellement à 34 exemples, pour les régions limitrophes de Río Bec (Figure 2). Plusieurs questions se posent donc pour les rares cas connus de la zone de Río Bec.

Une première évaluation de la répartition des terrains connus pour la région de Río Bec et les zones voisines permet de dénombrer 39 terrains répartis sur 36 sites, sans compter les reconstructions, comme à Becán (Figure 3).

\begin{tabular}{|c|c|c|c|c|c|}
\hline Site & Str. & Type & Allée & Datation & Référence principale \\
\hline Altamira & 3 & $\mathrm{I}$ & $14 \times 4$ & $\mathrm{CR}$ & Ruppert et Denison 1943 \\
\hline Altar de los Reyes & $16 a, b$ & $\mathrm{I}$ & & CR-CT? & Špracj et al. 2008 \\
\hline Balakbal & 11 & II & $29 \times 12$ & $\mathrm{CR} ?$ & $\begin{array}{l}\text { Ruppert et Denison } 1943 \text {; } \\
\text { Špracj et al. } 2008\end{array}$ \\
\hline Balamkú & & $\mathrm{NC}$ & $27 \times 8$ & $\mathrm{CR}$ & Michelet et al. 1998 \\
\hline Becán & 10 & I v1 & $32 \times 9.2$ & $\mathrm{CR}$ & Adams 1975 ; Bueno 1999 \\
\hline Calakmul & 11 & $\mathrm{I}$ & $29 \times 12$ & CR (731) & Carrasco 1996 \\
\hline Chactún & $?$ & & & & Špracj 2013 \\
\hline Chactún & ? & & & & Špracj 2013 \\
\hline Chakanbakan & & $\mathrm{NC}$ & & CR? & Cortes de Brasdefer 1997 \\
\hline Champerico & $11 \mathrm{a}, \mathrm{b}$ & II & & CA-CR & Špracj et al. 2008 \\
\hline Cheyokolnah & $6-7$ & $\mathrm{I}$ & $14 \times 6$ & CR-CT? & Špracj et al. 2008 \\
\hline Chicaanticaanal & 6 & $\mathrm{I}$ & & Pré-CA & Špracj et al. 2008 \\
\hline Dos Aguadas & 18 & $?$ & $20 \times 7$ & CR-CT? & Špracj et al. 2008 \\
\hline Dzibanché & & VI & $35.2 \times 11.2$ & CT & Nalda et al. 1994 \\
\hline Dzibanché & & VI & $32.6 \times 10.6$ & CT & Nalda et al. 1994 \\
\hline El Palmar? & & $\mathrm{I}$ & & $554-884$ & Špracj et al. 2008 \\
\hline El Tigre, Gr. A & $\mathrm{d} 1 / \mathrm{d} 2$ & II & $19 \times 50$ & $\mathrm{CT}$ & Vargas et Delgado 2003 \\
\hline
\end{tabular}




\begin{tabular}{|c|c|c|c|c|c|}
\hline El Zacatal & & II ? & & CR ? & Špracj et Esquivel 2010 \\
\hline Gruta de Alux & S2W1 (1-2) & $\mathrm{NC}-\mathrm{O}$ & $30 \times 10$ & $?$ & Normark 2009 \\
\hline Hopemul & $\begin{array}{l}\text { N1W1- } \\
\text { 4/N1E1-1 }\end{array}$ & NC-O & $20 \times ?$ & CR ? & Normark 2009 \\
\hline Kohunlich & $\mathrm{C} 1$ & I & $32.5 \times 12.5$ & CR & Cortés de Brasdefer 1998 \\
\hline Las Tuchas Bravas & & & & & Špracj et al. 2010 \\
\hline Los Alacranes & 7 & I & $35 \times 2.94$ & $\mathrm{CT}$ & Špracj et al. 2008 \\
\hline Los Angeles & & $\mathrm{I}$ & & $?$ & Špracj et al. 2008 \\
\hline Mucaancah & $14 a, b$ & I & $20 \times 8.5$ & $\mathrm{CR}$ & Špracj et al. 2008 \\
\hline Naachtun & & I v1 & $24 \times 5$ & CR ? & $\begin{array}{l}\text { Ruppert et Denison } 1943 \text {; } \\
\text { Reese-Taylor } \text { et al. } 2005\end{array}$ \\
\hline Nadzca'an & Gr.Chi'ik & $\mathrm{NC}$ & $13 \times 3$ & $\mathrm{CR}$ & Pescador 1998 \\
\hline Nadzca'an & Gr.Bec & VI & 21.523 .85 & $\mathrm{CT}$ & Pescador 1998 \\
\hline Nochebuena? & & $\mathrm{NC}$ & $20 \times ?$ & CR ? & Ruppert et Denison 1943 \\
\hline Oxpemul & $10-11$ & $\mathrm{I}$ & $20 \times 3$ & $731-771$ & $\begin{array}{l}\text { Ruppert et Denison } 1943 \text {; } \\
\text { Špracj et al. } 2008\end{array}$ \\
\hline Peor Es Nada & 6 & I & $21 \times 8.5$ & $\mathrm{CR}$ & Ruppert et Denison 1943 \\
\hline Pol Box & West group & $\mathrm{NC}-\mathrm{O}$ & $?$ & CR ? & $\begin{array}{l}\text { Esparza Olguín et Pérez } \\
\text { Gutiérrez } 2009\end{array}$ \\
\hline Puerto Rico & & $\mathrm{NC}$ & $20 \times 9$ & $?$ & Benavides 1996 \\
\hline Ramonal Quemado & S1W1 (5-6) & & $30 \times ?$ & CR ? & Normark 2009 \\
\hline Resbalón & 16 & $\mathrm{NC}$ & & CR ? & $\begin{array}{l}\text { López Camacho et } \\
\text { Tsukamoto } 2003\end{array}$ \\
\hline Río Azul & $\mathrm{C} 2-3$ & $\mathrm{NC}-\mathrm{O}$ & $17 \times 8.5$ & $\mathrm{CR}$ & Adams 1999 \\
\hline Río Bec II & 4 & $\mathrm{I}$ & $35 \times 9.7$ & DébutCR & Ruppert et Denison 1943 \\
\hline Río Bec V & 2 & I & $16 \times 6$ & DébutCR & Ruppert et Denison 1943 \\
\hline Uxul & 14 & I & $17 \times 6$ & $622-$ & $\begin{array}{l}\text { Ruppert et Denison 1943; } \\
\text { Ruz Lhuillier } 1945\end{array}$ \\
\hline Yaxnohcah & B-9 & $\mathrm{I}$ & & CR-CT? & Špracj et al. 2008 \\
\hline Yo'okop & N5W2 (6-7) & II ? & $30 \times 6$ & CR? & Normark 2009 \\
\hline
\end{tabular}

FIG. 3 - Liste des terrains dans la région de Río Bec (en gras) et les régions limitrophes.

Pour ce qui est de la zone de Río Bec, le total s'élève à quatre terrains probables ou confirmés pour trois sites (Figure 2). En effet, Peor es Nada (Figure 4a), doté d'édifices à tours et de crêtes faîtières, est, selon Nondédéo (com. pers. 2010), complètement de style Río Bec, mais se localise aux marges de la zone d'expansion maximale (Figure 1). La présence d'un terrain à Peor es Nada s'expliquerait de différentes manières : soit dans un contexte d'influence Río Bec, soit du fait d'autres apports, soit, enfin, en fonction d'une occupation plus ancienne, comme cela s'est produit pour Balamkú, Nadzca'an, Kohunlich, voire Calakmul, où l'influence Río Bec ne se manifeste qu'après des architectures plus proches des traditions du Petén (Michelet et al. 1998 ; Pescador 1998 ; Carrasco 1996 ; Nalda, in Shaw et Mathews 2005). Peor es Nada est d'ailleurs 
assez proche de Pechal, site dont l'architecture comporte des édifices de style Petén, à côté d'autres proches du style Chenes (Nondédéo et Lacadena 2007 ; Nondédéo, com. pers. 2010). Pour ce dernier site comme pour Peor es Nada, une configuration de plusieurs bâtiments en amphithéâtre (Figure 4b) évoque, formellement du moins, le groupe central de Balamkú (Figure 4c), voire la disposition des édifices de Kajtún (Figure 4d), une disposition qui ne se retrouve pas dans les sites de style Río Bec. Parmi les 39 terrains inventoriés, seuls ont été fouillés ceux de Becán, Calakmul, Kohunlich, Nadzca'an et Río Azul ${ }^{7}$. Ceux de Río Bec ont fait l'objet de simples sondages.

La Figure 4 présente les plans comparés de Peor es Nada, Pechal, de Balamkú (groupe Central) et de Kajtún avec leur disposition semi-circulaire.

Les recherches intensives restent peu nombreuses, et on manque cruellement de données sur des régions entières comme le Sud du Quintana Roo ou la partie occidentale de l'aire envisagée (au nord de Balamkú, par exemple). Des doutes subsistent aussi sur l'existence des terrains de Dos Aguadas et El Palmar ou de certains sites de la région de Cochuah (Normark 2009). Les estimations précédentes sont donc susceptibles d'évoluer, soit par l'identification de nouveaux terrains, soit en fonction d'une fouille qui infirmerait leur existence. Il est aussi nécessaire de tenir compte de la superficie des régions envisagées : l'aire Río Bec occupe un territoire plus restreint que l'ensemble des autres régions considérées. Il serait donc normal que le nombre de terrains y soit proportionnellement plus faible.

En corollaire, pour ce qui est des sites Río Bec, on doit reconnaître une insuffisance marquée des relevés de sites (Bueno 1999 ; Nondédéo 2003). Il est envisageable que de nouveaux terrains puissent être identifiés. Il serait cependant peu vraisemblable que leur nombre augmente dans des proportions suffisantes pour corriger la disproportion existante. Les travaux intensifs menés entre Becán et Chicanná (Eaton 1972; Thomas 1981), à Chicanná (Carrasco 1994), Hormiguero (Bueno 1999), Xpuhil (certes en partie détruit), Okolhuitz (Benavides 1997), Payán, ou dans le cadre du projet Manzana (Benavides 1996) n'ont abouti qu'à la seule identification, hypothétique, du terrain de Puerto Rico. Les propres recherches de Nondédéo (2003), bien qu'elles n'aient touché que partiellement des sites majeurs comme Tigre Triste ou Manos Rojas, et la reconnaissance intensive dans la micro-région autour de Río Bec n’ont pas permis d'identifier un seul terrain (Nondédéo, in Michelet et al. 2004, 2005a, 2006). Sur la base des données disponibles, il est probable que le nombre de terrains de la zone Río Bec stagne ou n'augmente que très peu, alors que pour les zones voisines, on enregistre un accroissement régulier. Sans compter la confirmation de l'existence de terrains déjà connus, les travaux de Špracj et al. (2008) dans le Sud-Est du Campeche ont accru de onze exemples un corpus auparavant réduit. Il est donc vraisemblable que la disproportion augmente 


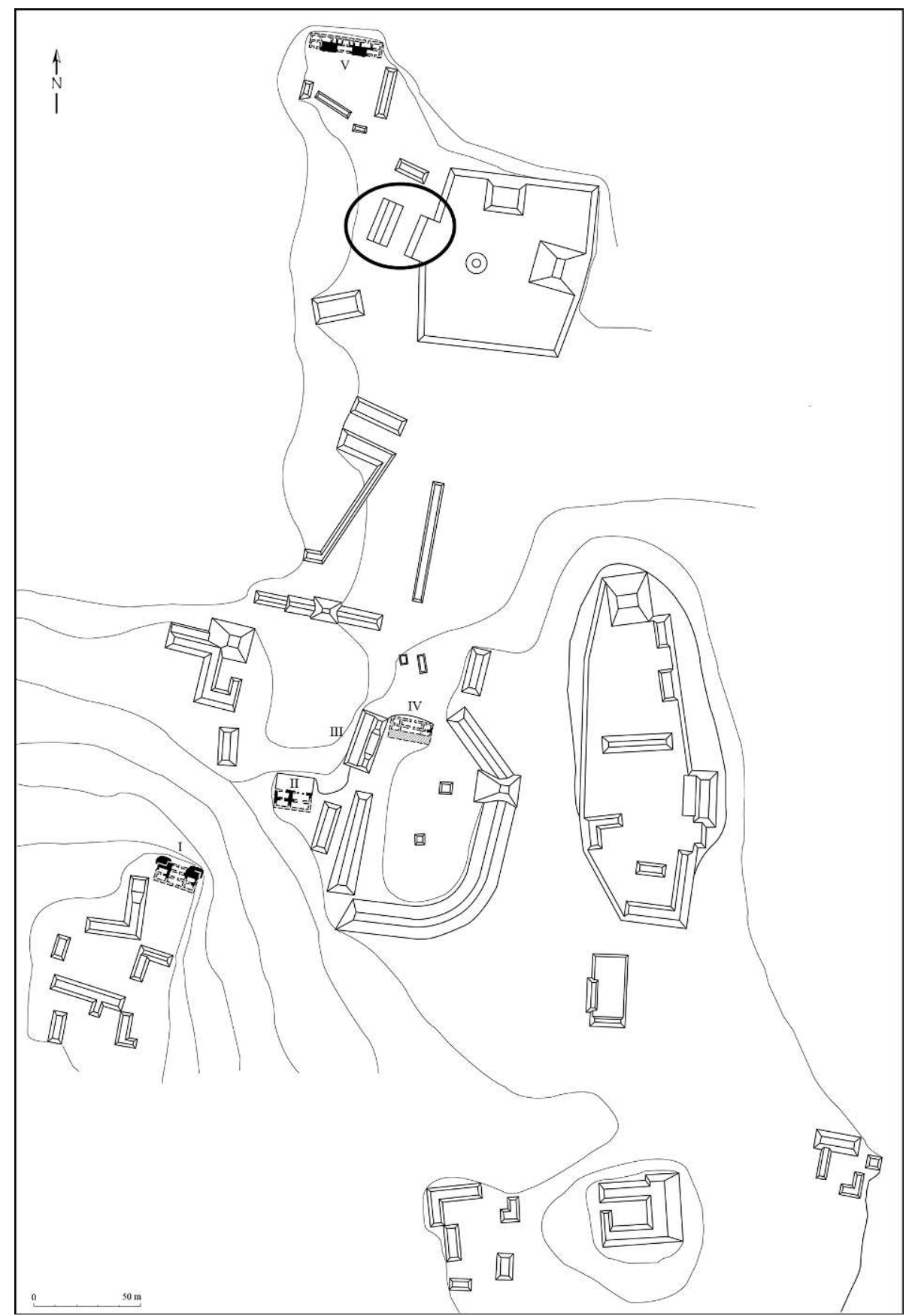

Fig. 4a - Peor es Nada (d'après Ruppert et Denison 1943), avec indication de l'emplacement du terrain de jeu de balle. 


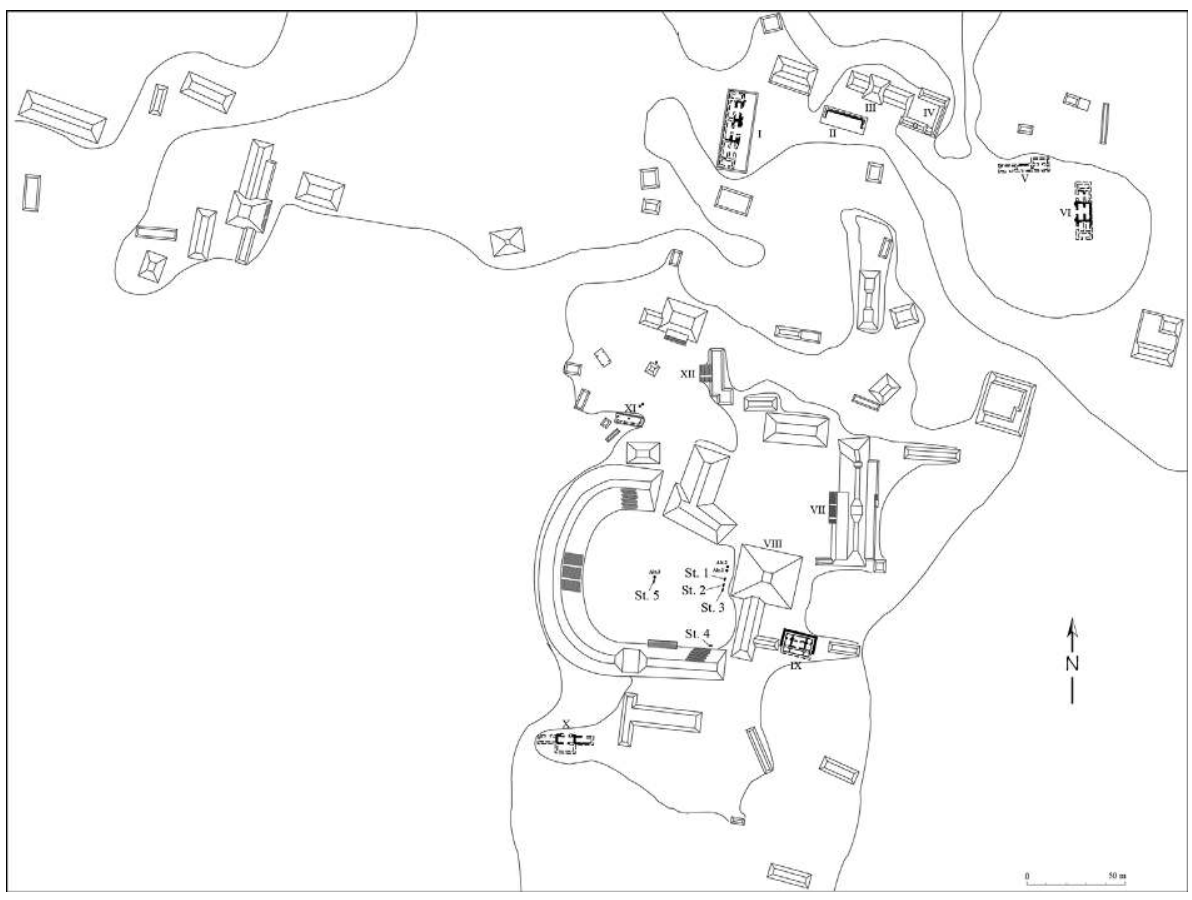

Fig. 4b - Pechal (d'après Ruppert et Denison 1943).

encore, au détriment de Río Bec (Figure 2). En réponse aux doutes exprimés par Nondédéo (2003), l'absence ou la rareté des terrains est bel et bien un caractère discriminant de la région Río Bec.

\section{IDENTIFICATION ET DATATION DES TERRAINS DE JEU DE BALLE}

Le deuxième volet de notre étude porte sur deux points complémentaires. D'abord, deux structures parallèles ne constituent pas forcément un terrain et, en l'absence de fouilles, un doute subsiste toujours sur l'identification définitive. Cet argument reste valable pour la quasi-totalité des exemples mentionnés dans la Figure 3, à l'exception des terrains fouillés, soit Becán, Río Azul, Calakmul, Kohunlich, Nadzca'an et Río Bec (Groupes II et V). Même si on peut raisonnablement valider la majorité des autres exemples, la prudence s'impose. C'est dans 
ce contexte que se sont situés les sondages des terrains des Groupes II et V de Río Bec. Ces groupes possèdent chacun un terrain ou, du moins, ce que Ruppert et Denison (1943) avaient identifié comme tel (Figures 5 et 6). Il importait donc de confirmer ou d'infirmer cette proposition et d'obtenir un maximum de données architecturales et chronologiques.

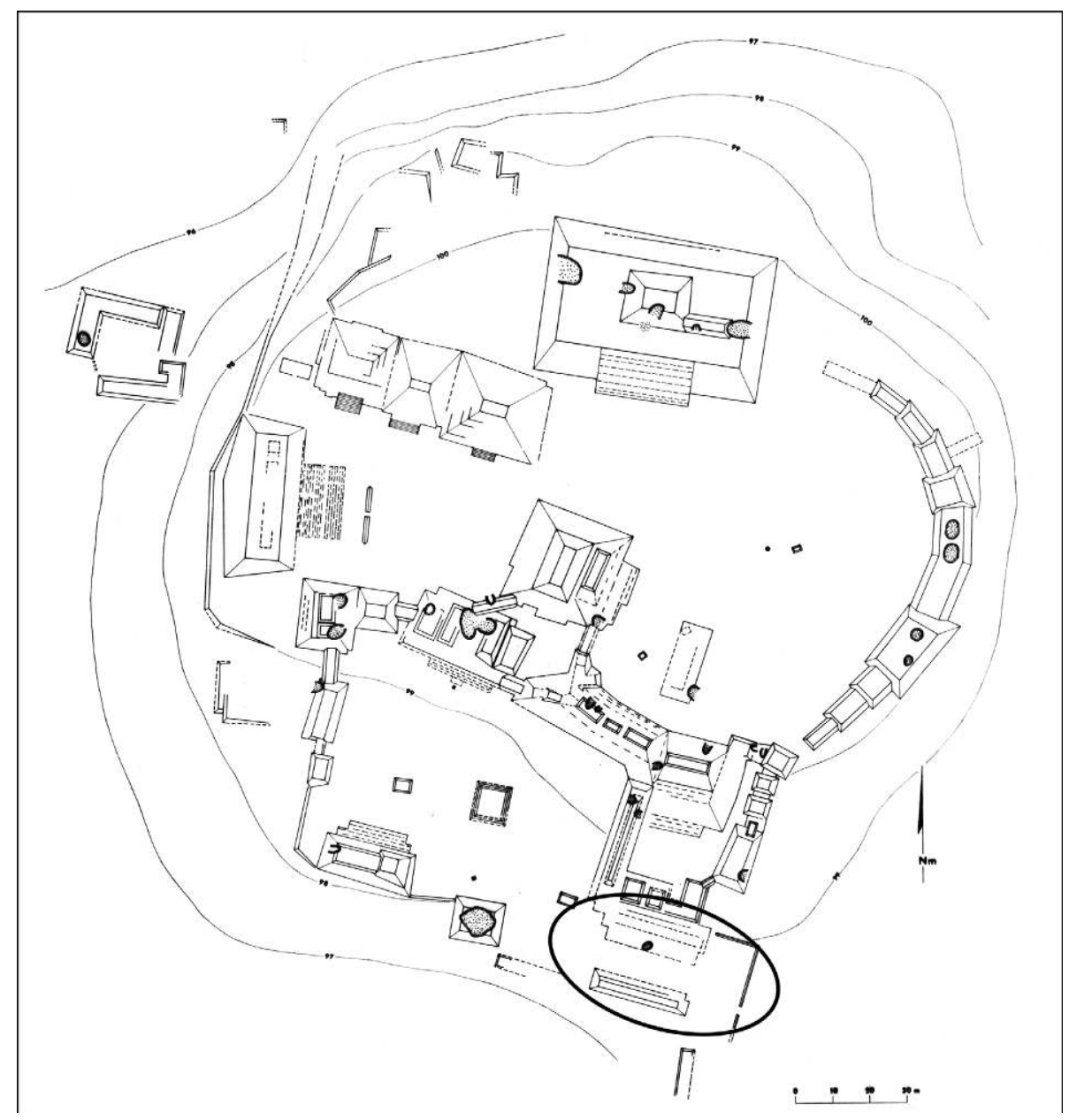

FIG. 4c - Balamkú (groupe Central), avec indication de l'emplacement du terrain de jeu de balle. Relevé de J.-P. Courau. 


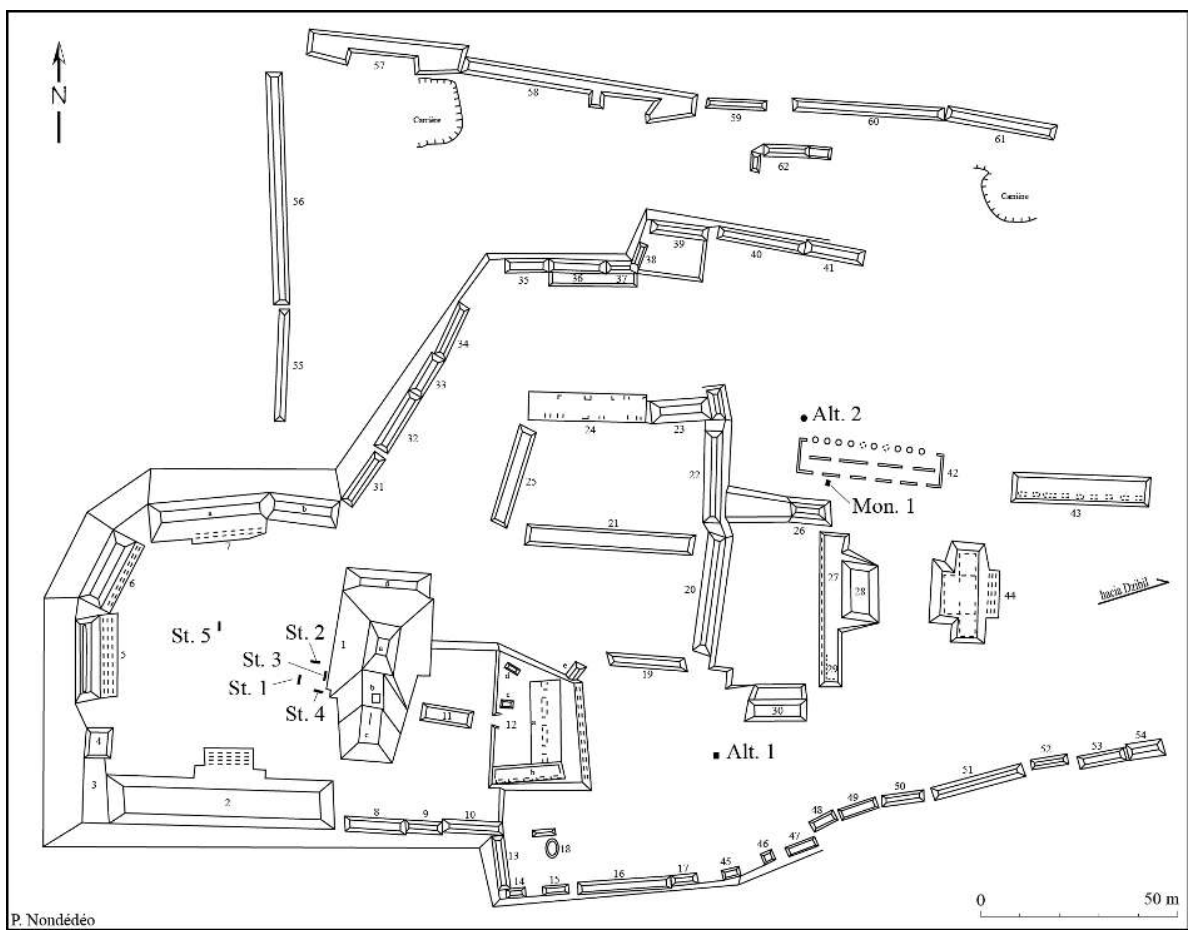

Fig. 4d - Kajtún (relevé de P. Nondédéo), avec indication des stèles.

Le terrain du Groupe II, la structure IV, se présente sous la forme de deux structures parallèles allongées avec une orientation générale de $283^{\circ}$ (Figure 5). La structure nord, d'une hauteur moyenne de trois mètres, est isolée, la structure sud est adossée à la pyramide (Str. III) du groupe, au sommet de laquelle est érigée la stèle 5, datée de 9.2.0.0.0., 475 apr. J.-C. (Lacadena 2004b, 2006). Après nettoyage, il a été possible d'identifier les angles sud-est et sud-ouest de la structure nord, ainsi que, plus hypothétiquement, l'angle nord-est de la structure sud. On a pu ainsi évaluer la longueur totale du terrain, entre 34,50 et $35 \mathrm{~m}$.

Si l'allée se présente comme un espace plan, il existe à son extrémité nordouest une cavité circulaire d'environ $1,50 \mathrm{~m}$ de profondeur pour 3 à $4 \mathrm{~m}$ de diamètre. À $5 \mathrm{~m}$ à l'ouest de l'angle sud-ouest, on enregistre la présence d'une meule incurvée. Ces deux éléments pourraient correspondre à des activités postérieures à l'abandon du terrain, que de rares indices céramiques dateraient de la phase Makan (700-850) (Taladoire et al. 2013). Il s'agirait d'une occupation 


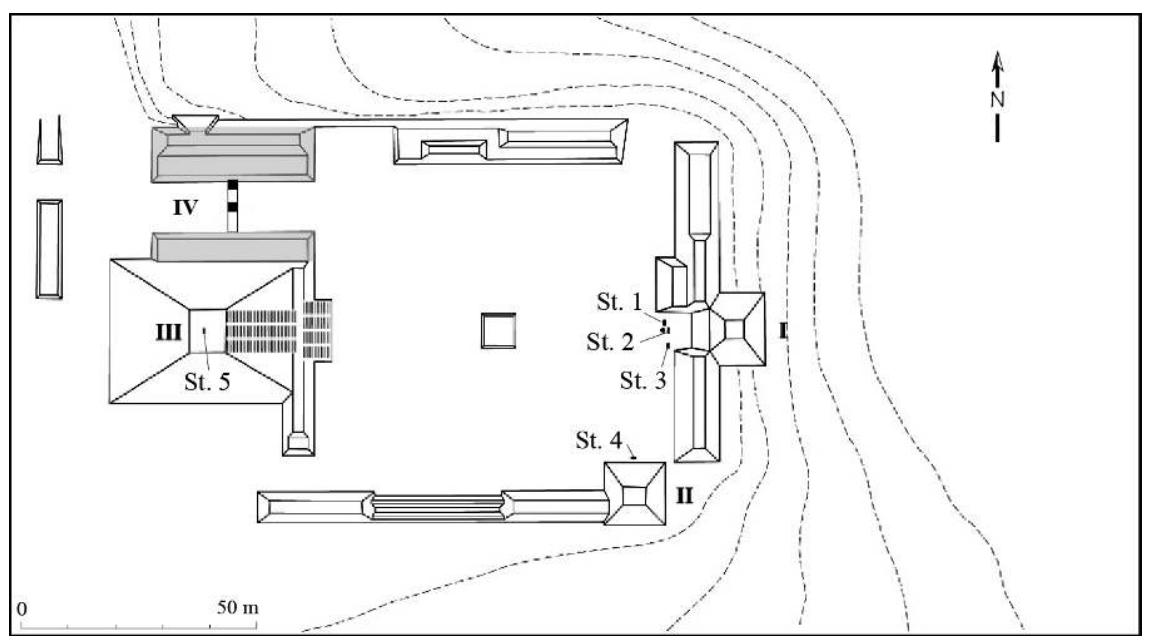

Fig. 5 - Plan du Groupe II de Río Bec, d'après Ruppert et Denison (1943), avec indication des stèles et des sondages effectués. Le terrain de jeu de balle est la Str. IV.

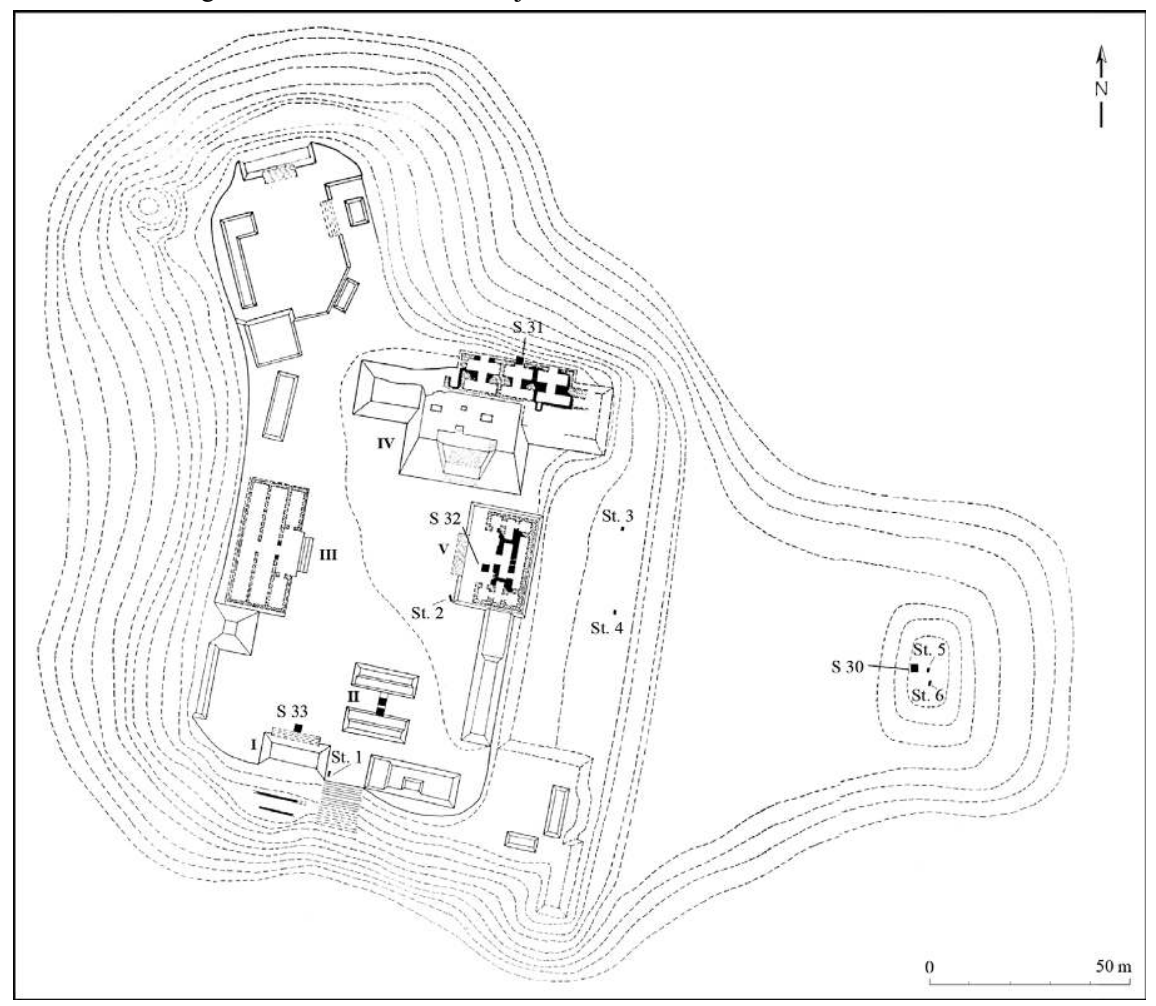

FIG. 6 - Plan du Groupe V de Río Bec, d'après Ruppert et Denison (1943), avec indication des stèles et des sondages effectués. Le terrain de jeu de balle est la Str. II. 
domestique, sans lien avec l'ancienne fonction du terrain. La fouille a établi le profil des structures latérales, confirmant ainsi l'identification du jeu de balle. L'allée mesure 34,50 à $35 \mathrm{~m}$ de long pour 9,70 $\mathrm{m}$ de large. Elle est délimitée par deux structures latérales en talus d'environ $27-28^{\circ}$, qui tombent sur l'allée par un bord vertical de $80 \mathrm{~cm}$ de haut. Les talus, assez étroits (entre 80 et $100 \mathrm{~cm}$ ), auraient été surmontés d'une corniche aujourd'hui disparue. La présence de blocs taillés dans les décombres permet d'inférer son existence et une hauteur minimale de $25 \mathrm{~cm}$. La morphologie des deux structures, le profil transversal et les dimensions confirment l'existence de ce terrain.

L'occupation du Groupe II commence au Préclassique moyen, avec une croissance durant la phase Bohom (300 av. J.-C.-300 apr. J.-C.). La construction des principaux édifices aurait eu lieu durant les phases Guayacan (300-425) et Iximché (425-550; ibid.), ce qui est compatible avec la date de la stèle 5 (Lacadena 2004b, 2006, 2007). L'occupation se poursuivrait jusqu'à la fin de la phase Iximché, avec en particulier la construction d'un autel au pied des stèles et celle du terrain de jeu de balle. Le matériel de remblai du terrain date sans exception des phases Bohom, Guayacan et Iximché. Une occupation ultérieure du groupe se manifeste par la présence, en quantité réduite (17 tessons soit 3,7\% du total recueilli dans le terrain de jeu de balle) de matériel des phases Makan (700-850) et Xpuhuk (850-950) (Dzul et Taladoire 2010), peut-être à relier aux vestiges d'occupation domestique mentionnés ci-dessus. Le terrain du Groupe II est donc daté d'Iximché, soit de la fin du Classique ancien.

Le terrain du Groupe V, la structure II, se présente sous la forme de deux structures parallèles allongées, d'une longueur estimée de $16 \mathrm{~m}$, séparées par un espace plan de $6 \mathrm{~m}$ de large (Figure 6). La hauteur moyenne des structures latérales est de $1,80 \mathrm{~m}$. L'orientation générale est de $293^{\circ}$, proche de celle du terrain du Groupe II. Les édifices se trouvent au sud d'une place, au sommet de l'élévation sur laquelle est implanté le groupe. Cette localisation pourrait paraître inhabituelle ${ }^{8}$ si Špracj et al. (2008) dans le Sud-Est du Campeche n'avaient pas enregistré plusieurs terrains occupant des emplacements similaires (Uxul, Mucancaah).

L'état global de destruction ne permet pas, en l'absence de fouilles extensives, d'être totalement affirmatif sur l'existence du terrain. Toutefois, plusieurs éléments convergent pour une identification positive.

- La morphologie générale et les proportions de l'allée sont conformes à celles d'un terrain.

- La hauteur des structures latérales est comparable à celle des structures du terrain du Groupe II, et leur étroitesse laisse peu de place pour y implanter des habitations.

- La similitude entre les deux exemples (espace plan central, formé de couches de remblai soigneusement superposées, rebord vertical du talus construit en dalles carrées) plaide dans le même sens. 
- Enfin, l'absence de toute autre fonction possible de ces structures est un argument complémentaire.

Il est donc probable que la structure II du Groupe V soit un terrain de jeu de balle. La question de sa datation reste, en revanche, complexe. Le matériel de remblai de l'allée (niveaux 3 et 4, sous un sol très détruit) est intégralement daté des phases Bohom, Guayacan et Iximché. On n'a trouvé que quatre tessons de la phase Makan, en surface. Dans le remblai effondré de la structure latérale nord, seuls trois tessons (sur 12) datant de la phase Makan ont été comptabilisés dans le niveau 1, de surface. Dans le niveau 2, l'ensemble du matériel (32 tessons) correspond à la phase Iximché. Il est donc tentant d'avancer une construction datant de la phase Iximché (425-550) ou Kanlol 1 (550-625), avec une possible occupation jusqu'en phase Makan.

Les autres sondages effectués dans le Groupe V par Nondédéo (in Michelet et al. 2005a) ont toutefois abouti à des conclusions différentes. En effet, l'analyse de la céramique des sondages exécutés a permis d'établir notamment que la place du groupe où se situe le terrain a été édifiée ou réaménagée à la fin du Classique récent comme l'attestent le matériel de cette période retrouvé dans le remblai du sondage S33 (Figure 6) et un dépôt rituel d'un encensoir de type Tecolote Compuesto, également daté de la phase Makan 2 (790-850). Dans le remblai du même sondage, du matériel en réemploi, s'étalant depuis le Préclassique récent jusqu'à la fin du Classique récent, domine l'échantillon. Le Classique terminal (phase Xpuhuk, 850-950), pour sa part, culmine à $15 \%$, soit l'un des pourcentages les plus élevés de l'ensemble des sondages.

L'occupation du Groupe V paraît donc relativement tardive, puisqu'au moins une partie de la place principale, celle où Nondédéo a effectué le sondage S33, a été construite ou refaite au Classique récent (phase Makan), et que la structure V, pour sa part, a été construite sans équivoque au tout début du Classique terminal (Xpuhuk). Les deux autres sondages de Nondédéo indiquent, eux aussi, une occupation du groupe légèrement antérieure au Classique terminal. Tel est le cas de la plate-forme aux stèles (S30) construite durant la seconde moitié du Classique récent (phase Makan), ce qui correspond à la datation épigraphique et stylistique des stèles 5 et 6 (Nondédéo et Patrois 2007), tandis que la structure IV (S31) est aussi datable du Classique récent. La faible quantité de matériel sous le sol de la pièce avant suggère une construction du soubassement de la structure IV à la transition entre le Classique ancien et le Classique récent. Mais selon l'architecture, l'édifice pourrait même dater de la seconde moitié du Classique récent (Makan 2). Dans un cas comme dans l'autre, l'occupation ne semble pas atteindre le Classique terminal, car aucun matériel de cette période n'a été retrouvé. Il existerait donc deux moments dans l'occupation du site, un premier dans la seconde partie du Classique ancien (phases Iximché et Kanlol) et un second au Classique récent et terminal (phases Makan et Xpuhuk). La construction du terrain pourrait dater de la première phase d'occupation du Groupe V, 
c'est-à-dire de la fin du Classique ancien, ce qui serait quasi identique à celle du terrain du Groupe II ${ }^{9}$.

Pour les terrains de Río Bec, la construction initiale de celui du Groupe II se serait produite en phase Iximché (425-550), avec une réfection éventuelle en Kanlol 1 (550-625). Celui du Groupe V aurait été édifié à la fin de la phase Iximché (425-550), mais plus probablement durant la phase Kanlol 1 (550-625) sur une terrasse aménagée antérieurement. On ne saurait affirmer une utilisation du terrain durant la phase Makan. Le terrain de Becán (structure XI) est daté du Classique récent. Durant les fouilles de 1969, l'équipe de Tulane University a identifié en surface les vestiges d'un marqueur central, brisé en plusieurs morceaux et porteur de motifs. Sous ce marqueur, une cache a livré une figurine du type Paaktzatz Modeled (Ball 1977, fig. 38-C), qui confirme la datation du Classique récent (Thomas, com. pers. $2007^{10}$ ). La présence du marqueur et de la cache suggère, par ailleurs, le caractère exceptionnel de ce terrain, car il s'agit, pour la région de Río Bec, d'un cas unique qui renvoie plutôt à des pratiques enregistrées dans les sites du Petén. Si l'existence de ces trois terrains est donc confirmée, en laissant de côté les exemples non datés de Peor es Nada et Puerto Rico, se pose la question de leur appartenance au style Río Bec, au sens large où nous l'entendons. En effet, leur localisation au sein de sites ou de groupes qui ont connu soit une occupation antérieure à l'apogée Río Bec (Groupe II et, en partie, le Groupe V), soit comme à Becán, une histoire plus complexe propre à un véritable centre, soulève la question de leur utilisation durant l'apogée Río Bec.

Pour la plupart des terrains localisés dans les zones limitrophes, le manque de datation se fait aussi cruellement sentir. Beaucoup n'ont pas été fouillés : leur datation n'est donc qu'estimée. C'est le cas de la majorité des terrains identifiés dans le Sud-Est du Campeche (Špracj et al. 2008), mais aussi celui de ceux de Dzibanché ou Balamkú (Figure 3). En fonction des données disponibles, la majorité daterait du Classique récent ou, au plus tôt, de la fin du Classique ancien. Parmi les exemples fouillés, les terrains de Calakmul (Carrasco 1996), Kohunlich (Nalda, in Shaw et Mathews 2005), Nadzca'an (Groupe Chi'ik ; Pescador 1998), Río Azul (Adams 1999) et peut-être Oxpemul (Folan et al. 2005) datent en tout cas du Classique récent. On peut aussi faire état de datations estimées du Classique terminal pour les exemples de Dzibanché (Nalda et al. 1994), El Tigre (Vargas et Delgado 2003) ou Nadzca'an (Groupe Bec ; Pescador 1998). Si ces deux derniers sites comportent des traits et des édifices Río Bec, ils sont localisés à l'extérieur de la région éponyme. La construction tardive de leurs terrains correspondrait à une phase au cours de laquelle le style Río Bec tendrait à s'estomper. L'édification de terrains au Classique terminal pourrait alors s'interpréter comme un événement local (Dzibanché), un retour à des pratiques antérieures à l'introduction du style (El Tigre, Nadzca'an) ou un indice de l'apparition d'influences septentrionales, très fortes dans la région (Nondédéo et Lacadena 2004 ; Nondédéo et Patrois 2007). Ces exemples tardifs 
ne peuvent en aucun cas être associés à une manifestation ou à une influence du style Río Bec.

Pour l'ensemble de la région considérée, la pratique du jeu est donc attestée depuis au moins la fin du Classique ancien, et tout au long du Classique récent jusqu'au Classique terminal dans les sites des régions limitrophes. D'autres éléments, comme des représentations de joueurs, à Mucaancah (Špracj, García Cruz et Ojeda Mas 1997 ; Špracj et al. 2008) ou à Ichmul (Lacadena 2004a) renforcent cette proposition.

On peut donc contraster deux situations distinctes. D'une part, une édification continue, tout au long du Classique récent, de terrains dans des centres en pleine activité, aux alentours de la région de Río Bec. D'autre part, une construction minimale sur quelques sites ou groupes de la région éponyme, dont deux sont d'ailleurs datés du tout début du phénomène Río Bec (Río Bec II et V), un autre est atypique (Becán), un dernier se situe en périphérie (Peor es Nada). Seul demeure en suspens le cas, non confirmé, de Puerto Rico. Sauf pour Becán et peut-être Puerto Rico, on doit donc conclure à une extrême rareté du jeu durant l'apogée du phénomène Río Bec.

\section{STÈLES ET INSCRIPTIONS}

Il n'est pas question d'envisager ici une approche épigraphique qui ressort d'un autre travail (Lacadena 2004b, 2006, 2007). L'étude porte sur la présence de stèles et de monuments sculptés, représentatifs d'une manifestation du pouvoir dynastique (Figure 2). Le déchiffrement des textes est susceptible de révéler l'existence ou la nature d'un pouvoir local ou régional (Nondédéo et Lacadena 2004 ; Mayer 2009 ; Nondédéo et al. 2013), ce qui peut modifier, voire contredire certaines de nos hypothèses. C'est avant tout la rareté des monuments dans la région Río Bec qui attire l'attention, et implique, globalement, l'hypothèse d'un pouvoir différent, quitte à ce que la lecture des rares inscriptions soulève la question de variations locales ou d'évolutions possibles. Si les stèles sont l'objet prioritaire de notre attention, ce ne sont pourtant pas les seuls supports de l'idéologie dynastique. Sur des sites du Sud du Campeche comme Altar de los Reyes ou Oxpemul, des inscriptions figurent aussi sur des autels (Špracj et al. 2008). D'autres sites, s'ils ne comptent que peu ou pas de stèles, n'en sont pas moins pourvus d'inscriptions sur des contremarches ou des panneaux à El Resbalón (Carrasco et Boucher 1987 ; Beliaev et Safronov 2002) et Dzibanché (Nalda et al. 1994). Á Mucancaah (Špracj et al. 2008), les panneaux peuvent n'être qu'une variante locale, puisque le site voisine avec d'autres qui comptent des stèles (El Palmar, Los Alacranes). En revanche, pour El Resbalón et Dzibanché, comme pour l'ensemble des sites de cette partie du Quintana Roo (Chakanbakan et Kohunlich), la rareté des stèles avec des inscriptions reste 
inexpliquée. Kohunlich ne possède que des stèles lisses. D'autres stèles lisses ont été enregistrées à Calakmul, Nadzca'an (stèle 21), Becán (1, 2, 5, 6, 7, 11), Manos Rojas 3, Pechal (stèle 5) ou Kajtún (stèle 5). Toutefois, la découverte sur le site de Pol Box de plusieurs monuments sculptés avec des inscriptions (Esparza 2006 ; Esparza Olguín et Pérez Gutiérrez 2009) permet d'espérer une augmentation future du corpus. Qu'il s'agisse de stèles, d'autels ou d'autres supports, nombre de sites de cette partie du Quintana Roo comptent donc des inscriptions hiéroglyphiques (El Resbalón, Dzibanché, Pol Box, Chacchoben) et participent de la tradition Petén. D'autres sites plus septentrionaux entrent également dans cet ensemble comme Yo'okop, avec deux stèles et une représentation de joueur (Wren et Nygard 2005) ou Chichmuul (Harrison 2005). Harrison signale aussi que de nombreux monuments sculptés auraient disparu de cette partie du Quintana Roo, avant leur enregistrement. L'identification d'une éventuelle sculpture de Dzibanché au musée de Cancún, datée de 9.6.0.0.0. (554) en serait un exemple (Mayer 1982).

En l'état actuel des connaissances, donc, et comme pour les terrains, le contraste est marqué entre la région de Río Bec et les régions limitrophes. Sans considérer les sites mineurs du Sud-Est du Campeche qui ne comptent qu'un ou deux monuments (Grube, in Špracj et al. 2008), le nombre de sites comportant des monuments porteurs d'iconographie ou d'inscriptions s'élève à 42, dont 12 seulement dans la région Río Bec. Le nombre de monuments enregistrés pour les sites Río Bec atteint désormais 44 exemplaires dont 22 lisses. Face aux 117 stèles du seul site de Calakmul et au nombre élevé de monuments sur des sites comme El Palmar (44), Oxpemul (19) ou Uxul (16) ce total paraît très réduit (Figure 7). Il l'est plus encore si l'on considère qu'une partie des monuments de Becán et au moins une stèle du Groupe II de Río Bec précèdent l'apogée du phénomène Río Bec. De plus, les cinq monuments de Pechal se trouvent sur un site localisé aux confins de la zone d'expansion maximale du style et qui connaît une influence du style Chenes. Selon Proskouriakoff (1950), le style des inscriptions de Pechal aurait une origine plus septentrionale (Bueno 1999), ce que confirment Nondédéo et Lacadena (2007). En l'absence de déchiffrement, on peut donc supposer qu'ils doivent être, sinon exclus du corpus, du moins considérés à part. D'autres aspects de la sculpture dans l'aire Río Bec montrent d'ailleurs des indices d'influences ou d'apports venus du Yucatan (Nondédéo et Lacadena 2004; Nondédéo et Patrois 2007). Le corpus de monuments dans la région de Río Bec se réduit donc à 39 exemples de Pasión del Cristo, Río Bec proprement dit, Kajtún, Dzibil et Becán. 


\begin{tabular}{|c|c|c|c|c|}
\hline Sites & Stèles & Mon. & Fourchette de datation & Référence \\
\hline Altamira & 17 & $\mathrm{X}$ & $?$ & Grube (in Špracj et al. 2008) \\
\hline Altar de los Reyes & 2 & $\mathrm{X}$ & 800 (St. 1)- & Grube (in Špracj et al. 2008) \\
\hline Balakbal & 4 & & 386 (St. 5)-406 (St. 5) & Grube (in Špracj et al. 2008) \\
\hline Balamkú & 4 & & & $\begin{array}{l}\text { Nondédéo } 2003 \text {; Michelet } \\
\text { et al. } 1998\end{array}$ \\
\hline Becán & 11 & $\mathrm{X}$ & $?$ & $\begin{array}{l}\text { Adams } 1975 \text {; Campaña } 2005 \text {; } \\
\text { Thomas } 1981\end{array}$ \\
\hline Calakmul & 117 & & 435 (St. 114)-810 & Carrasco 1996 \\
\hline Candzibaantun & 4 & & 396 (St. 2)-396 (St. 4) & Grube (in Špracj et al. 2008) \\
\hline Chacchoben & 1 & & $?$ & \\
\hline Chactún & 19 & & & Špracj 2013 \\
\hline Chakanbakan & $\mathrm{X}$ & $\mathrm{X}$ & $?$ & Cortes de Brasdefer 1994 \\
\hline Champerico & 4 & & 366 (St. 2)-613 (St. 1) & Grube (in Špracj et al. 2008) \\
\hline Cheyelkolnah & 1 & & 420 & Grube (in Špracj et al. 2008) \\
\hline Dos Aguadas & 2 & & $?$ & Grube (in Špracj et al. 2008) \\
\hline Dzibanché & & $\mathrm{X}$ & 909 & Nalda et al. 1994 \\
\hline Dzibil & 2 & & & Nondédéo et Lacadena 2004 \\
\hline El Coyote & 1 & & & $\begin{array}{l}\text { Nondédéo et Patrois } 2007 \text {; } \\
\text { Andrews } 1999\end{array}$ \\
\hline El Palmar & 44 & & 554 (Alt. 1)-820 (St. 14) & Grube (in Špracj et al. 2008), \\
\hline El Zacatal & $?$ & & & Špracj et Esquivel 2010 \\
\hline Kajtún & 5 & & $731-795$ & Nondédéo et Lacadena 2004 \\
\hline Kohunlich & $3+$ & $\mathrm{X}$ & $?$ & Cortés de Brasdefer 1998 \\
\hline La Muñeca & 19 & & 780 (St. 5)-889 (St. 1) & $\begin{array}{l}\text { Ruppert et Denison 1943; } \\
\text { Grube (in Špracj et al. 2008) }\end{array}$ \\
\hline Los Alacranes & 2 & & 504 (St. 1)-561 (St. 1) & Grube (in Špracj et al. 2008) \\
\hline Los Angeles & 15 & $\mathrm{X}$ & $?$ & Grube (in Špracj et al. 2008) \\
\hline Los Hornos & $?$ & & & Špracj et al. 2010 \\
\hline Manos Rojas 3 & 3 & & & Nondédéo 2003 \\
\hline Mucancaah & & $\mathrm{X}$ & $?$ & Grube (in Špracj et al. 2008) \\
\hline Naachtun & 48 & $\mathrm{X}$ & $?$ & $\begin{array}{l}\text { Ruppert et Denison } 1943 \text {; } \\
\text { Reese-Taylor } \text { et al. } 2005\end{array}$ \\
\hline Nadzca'an & $21+$ & & -830 & Pescador 1998 \\
\hline Oxpemul & 19 & $\mathrm{X}$ & 731 (St. 12)-830 (St. 7) & Grube (in Špracj et al. 2008) \\
\hline Pasión del Cristo & 3 & & $889-899$ & $\begin{array}{l}\text { Nondédéo } 2003 \text {; Bueno } 1999 \text {; } \\
\text { Proskouriakoff } 1950 \text {, fig. } 75 \text { a }\end{array}$ \\
\hline Pechal & 5 & & Classique terminal? & $\begin{array}{l}\text { Nondédéo et Lacadena } 2007 \text {; } \\
\text { Bueno } 1999 \text {; Ruppert et } \\
\text { Denison 1943, pl. 60f. }\end{array}$ \\
\hline Peor es Nada & 1 & & & Nondédéo, com. pers. \\
\hline Pol Box & 5 & & $573-593$ & $\begin{array}{l}\text { Esparza 2006; } \\
\text { Pérez Gutierrez } 2009\end{array}$ \\
\hline Pueblo Viejo B & 1 & & & Nondedéo et Patrois 2007 \\
\hline
\end{tabular}




\begin{tabular}{|l|c|c|l|l|}
\hline Resbalón & & X & $529-580$ & $\begin{array}{l}\text { Carrasco et Boucher 1986; } \\
\text { Beliaev et Safronov 2002 }\end{array}$ \\
\hline Río Azul & $2+$ & & $?$ & Adams 1999 \\
\hline Rio Bec II & 5 & & $475-672$ & Lacadena 2004b, 2006 \\
\hline Río Bec V & 6 & X & 613 ou 869 & Lacadena 2004b, 2006 \\
\hline Río Bec B & & X & 805 & Lacadena 2004b, 2006 \\
\hline San Lorenzo & 1 & & 790 (ou 928 ?) & $\begin{array}{l}\text { Thompson 1953; Nondedéo } \\
\text { et Patrois 2007 }\end{array}$ \\
\hline Uxul & 16 & $\mathrm{X}$ & 602 (Alt. 2)-692 (St. 14) & Grube in Špracj et al. 2008) \\
\hline Xpuhil VIII & 1 & & & Mayer 2003 \\
\hline Yo'okop & 2 & $\mathrm{X}$ & $?$ & Flores et al. 2008 \\
\hline
\end{tabular}

FIG. 7 - Répartition des monuments sculptés dans la région de Río Bec (en gras) et les secteurs voisins.

Inversement, on ne saurait négliger la possibilité de l'existence d'inscriptions sur d'autres supports ou sous-estimer leur apport. Le manque de fouilles intensives ne permet pas de trancher, même si les recherches à Chicanná, Xpujil, Becán ou Hormiguero n'ont pas donné lieu à de nombreuses découvertes (Bueno 1999 ; Mathews 1983 ; Mayer 1988, 2009). On peut pourtant faire état de l'identification de quelques glyphes peints à Hormiguero et à Chicanná (Str. II), ainsi que d'une pierre de voûte sur le même site (Str. IV) avec une date de 539 ou 591, et d'une autre pierre de voûte à Becán (Mathews 1983). D'autres vestiges de glyphes peints, aujourd'hui disparus, ont été signalés à Xpujil II (Str. 1) et à Becán (Str. IV ; Bueno 2001). Enfin, dans l'édifice 6N-2 de Río Bec, les fouilles ont dégagé des bords de banquettes porteuses d'inscriptions (Arnauld, in Michelet et al. 2005a, 2006), dont le déchiffrement a permis à Lacadena (2004b, 2006, 2007) d'obtenir une date correspondant à 805 apr. J.-C., dans un enregistrement en compte court sur le rebord de la banquette nord (Arnauld et Lacadena 2004). Reste qu'en l'état actuel des connaissances, cette découverte constitue un cas isolé. Il est donc possible, mais peu probable, que le nombre d'inscriptions augmente encore un peu. Les fouilles extensives des Groupes A et D de Río Bec n'ont pourtant pas révélé d'autres inscriptions.

Face à cette pauvreté de monuments sculptés et d'inscriptions dans le cœur de l'aire Rio Bec, à l'apogée du phénomène, l'abondance de stèles dans les sites voisins ressort d'autant plus nettement. Pas moins de 28 sites majeurs ou mineurs en comptent un total de 340, sans compter les contremarches et panneaux de Dzibanché, Mucaancah ou Resbalón. Même si la région de Río Bec couvre une superficie plus réduite que celle de la totalité des régions limitrophes, le contraste n'en demeure pas moins frappant.

Le phénomène est amplifié par l'examen des dates relevées. D'abord, une partie, certes limitée, des dates enregistrées sur des monuments de la région de Río Bec (Lacadena 2004b, 2006, 2007) est antérieure à l'apogée du style, ce qui 
conduit à exprimer des doutes sur la pertinence de leur inclusion dans le corpus. Le même doute porte sur la pierre de voûte de Chicanná avec sa date de 539 ou 591 (Mayer 1988). Certes, plusieurs sites voisins (Balakbal, Champerico, Los Alacranes...) n'ont donné que des dates du Classique ancien, ce qui peut traduire un ralentissement, au moins régional, de la pratique d'érection de monuments et, consécutivement, des changements politiques ou sociaux. Cela pourrait aussi traduire des changements des lieux de pouvoir, en liaison avec l'histoire régionale (Nondédéo et al. 2010). Par ailleurs, le site de Pechal se situe aux confins de la zone d'apogée et, même si l'occupation Río Bec du site est attestée (Nondédéo et Lacadena 2007), cette présence pourrait se substituer à une occupation antérieure. Il n'est donc pas certain que les stèles de Pechal s'intègrent véritablement au phénomène Río Bec.

Il est pourtant indéniable que l'érection de monuments sculptés se poursuit, sur la plupart des sites connus, simultanément au développement du style Río Bec. La plupart des sites voisins érigent des monuments tout au long du Classique récent. Citons les monuments de Calakmul, mais aussi ceux d'Altar de los Reyes, El Palmar, La Muñeca, Oxpemul et Uxul (Grube, in Špracj et al. 2008), voire plus tardivement au Classique terminal, comme le suggèrent les dates de Dzibanché (Nalda et al. 1994). Certains groupes Río Bec se dotent de stèles dont le déchiffrement confirme un placement au Classique récent: la stèle $3 \mathrm{du}$ Groupe II (9.12.0.0.0. 10 Ajaw 18 Yaxkin soit 672), la stèle 4 du Groupe V (9.9.?.?? soit 613 ou 633), les stèles 4 de Kajtún (9.15.0.0.0. 14 Ajaw 13 Yax soit 731) et 6 de Dzibil (9.18.5.0.0. soit 795), les stèles de Pasión del Cristo (10.3.0.0.0 soit 889), la stèle 1 de San Lorenzo datée de 10.5.0.0.0 10 Ajaw 8 Muan, 928 selon Thompson (1953) ou selon Mayer (2003) de 9.18.0.0.0, 11 Ajaw 8 Muan (790). Les banquettes de la structure $6 \mathrm{~N}-2$ donnent la date de 805. Par ailleurs, l'analyse iconographique et stylistique menée par Patrois, en fonction de l'étude de Proskouriakoff (1950), autorise une datation préliminaire de la phase dynamique (entre 750 et 800 apr. J.-C.) de six monuments supplémentaires, soit les stèles 5 et 6 du Groupe V, la stèle 3 de Kajtún et probablement les stèles 1, 2 et 3 de Pechal (Nondédéo et Patrois 2007). À l'image des monuments du Groupe V, certaines stèles de Kajtún pourraient relever du même phénomène de recours, tardif, aux monuments sculptés.

Il serait donc absurde de penser que l'élite dirigeante de la région de Río Bec ait ignoré cette pratique d'érection de monuments sculptés. Elle est connue dans le cœur de l'aire, et lors de leur installation sur des sites comme Nadzca'an (Pescador 1998), Balamkú (Michelet et al. 1998) ou Calakmul (Carrasco 1996), les porteurs du style Río Bec ont été confrontés directement à leur présence. La connaissance et l'usage de l'écriture sont également attestés par les monuments de Becán, Río Bec, Kajtún ou les inscriptions peintes. Il est donc impossible de nier que la rareté des monuments sculptés et des inscriptions glyphiques, à l'instar de celle des terrains de jeu de balle, ne relève pas d'un choix délibéré. 


\section{PourQuoI NI TERRAINS, NI STÈLES À INSCRIPTIONS ?}

Longtemps, monuments sculptés porteurs d'inscriptions et terrains de jeu de balle ont été considérés comme les critères de définition d'un centre cérémoniel majeur (e.g. Bullard 1960). L'absence de l'un ou de l'autre, voire des deux, reléguait un site à un rang secondaire. Nous ne reviendrons pas ici sur cette conception qui a un peu vieilli : l'affirmation d'un lien entre inscriptions, jeu et importance d'un site demeure malgré tout globalement valide, même si ce lien n'est pas systématique : ainsi, plusieurs centres politiques mayas sont apparemment dépourvus de terrains. On peut citer Bonampak, Ichmul, Motul de San José ou La Joyanca (Arnauld, Breuil-Martínez et Ponciano Alvarado 2004). Inversement, des terrains ont été identifiés sur des sites mineurs (Petultón dans la vallée d'Ocosingo ou El Tambo près de La Joyanca), voire franchement petits (Mosil B ; Becquelin et Taladoire 1990 ; Taladoire 1980; Arnauld, BreuilMartínez et Ponciano Alvarado 2004). De même, Špracj et al. (2008) en enregistre plusieurs dans des sites secondaires du Sud-Est du Campeche, dont Candzibaantun ou Chicaanticaanal.

Il en va de même pour les stèles et autres monuments, puisque, dans cette même région du Campeche, Špracj et al. (2008) ont relevé la présence de monuments isolés sur de petits sites (Las Tuchas). Il est donc possible, pour des sites de petite taille, voire pour des groupes comme ceux de la zone de Río Bec, d'affirmer leur identité en érigeant ces symboles de pouvoir. La présence de stèles et de terrains dans les Groupes II et V de Río Bec (Michelet et al. 2008), ainsi que les banquettes de la structure 6N-2 (Arnauld et Lacadena 2004) en sont de bons exemples, tout autant que les quelques cas cités par Bueno (1999). La rareté de ces traits dans les groupes Río Bec ne peut donc se justifier par le seul critère de leur taille ou de leur importance.

Une démarche différente conduirait à considérer séparément chaque trait. Au Postclassique récent, au Yucatan, le jeu n'est plus pratiqué et des sites comme Mayapan, Muyil ou Tulum sont dépourvus de terrain (Taladoire 1981). Inversement, l'écriture demeure hautement significative, même si les monuments sculptés laissent place à d'autres supports, les codices ou les peintures murales, à Tulum ou Santa Rita par exemple. Nous sommes ici confrontés à une dissociation de ce qui était considéré comme une conjonction. Toutefois, dans ce cadre précis, l'écriture a perdu une part de son caractère politique et historique pour revêtir un rôle plus religieux et divinatoire, même si ces deux aspects restent inextricables. Cette évolution du politique vers le rituel, qui s'applique à l'écriture, trouve son pendant pour le jeu, dont le rôle et la fonction politique ne se justifient plus. La disparition du pouvoir dynastique a oblitéré la responsabilité du pouvoir, telle qu'elle s'exprimait par le jeu ${ }^{11}$. 
Toujours selon la même démarche, mais dans un autre contexte, la pratique du jeu s'est perpétuée parmi des cultures qui n'ont pas fait le même usage que les Mayas des textes écrits. Elle atteint des sommets dans les civilisations sans écriture de la côte du Golfe, avec les 17 terrains d'El Tajín (Raesfeld 1990, 1992) ou les 25 de Cantona (García Cook et Merino Carríon 1998). De même, chez les Mexicas, le jeu est indubitablement associé au pouvoir, alors que l'écriture occupe une place bien différente. Il n'est donc ni nécessaire, ni indispensable de lier les deux traits qu'il serait possible, au contraire, de dissocier. Dans cette perspective, l'absence simultanée de l'un et de l'autre sur les sites Río Bec pourrait n'être que fortuite. Cette explication paraît toutefois peu vraisemblable pour de multiples raisons. La plus évidente est l'omniprésence dans les régions avoisinantes des deux traits, en abondance. Leur absence simultanée dans la plupart des sites Río Bec ne se justifie donc en aucune manière par une méconnaissance, d'autant moins qu'en dépit de leur rareté, terrains et monuments sculptés existent bel et bien sur des sites Río Bec.

Mais surtout, on ne peut nier le lien étroit qui unit, chez les Mayas, jeu de balle et écriture, à travers l'exercice du pouvoir dynastique. Les stèles et les autres monuments sont l'expression même de ce pouvoir (Martin et Grube 2000). La pratique du jeu est l'une des responsabilités des dirigeants, que ce soit pour des raisons agraires à Copán (Baudez 1984) ou guerrières et sacrificielles à Yaxchilan (García Moll 1977), Toniná (Becquelin et Taladoire 1990) ou Cobá (Con Uribe 2000). L'articulation étroite entre ces deux expressions du pouvoir est attestée par l'iconographie et l'épigraphie. Nombre de monuments porteurs d'inscriptions et d'images de dirigeants (disques, stèles, panneaux) proviennent directement des terrains et l'iconographie du jeu figure sur d'autres édifices, dont l'escalier hiéroglyphique de Yaxchilan (García Moll 1977). Les glyphes du terrain et du jeu figurent de même sur de nombreuses inscriptions dynastiques (Boot 1991). Le lien entre ces deux traits est donc indéniable. Même dans notre zone d'étude, il est affirmé sur le site de Mucancaah (Špracj, García Cruz et Ojeda Mas 1997). On en retrouve une expression plus au nord, à Yo'okop (Wren et Nygard 2005) ou à Cobá (Con Uribe 2000). Leur point de conjonction se situe évidemment dans la nature même du pouvoir dynastique qui s'exprime simultanément sous ces deux formes. La dissociation proposée ci-dessus, pour d'autres civilisations ou d'autres périodes, ne saurait donc s'appliquer ici. L'existence de terrains et d'inscriptions dans des sites qui ont connu une manifestation du style Río Bec, au Classique récent, va d'ailleurs à l'encontre de cette hypothèse et prouve au contraire que le lien demeure. Á Río Bec même, la conjonction de la présence de stèles et de terrains dans les Groupes II et V démontre la connaissance de ce type de revendication dynastique (Nondédéo et al. 2013). L'absence manifeste des stèles et du jeu dans l'ensemble des sites Río Bec ne saurait donc se justifier par une circonstance fortuite, ni par une simple évolution de chaque trait vers une signification distincte, particulière à une expression régionale. 
Sous réserve donc de découvertes inattendues et peu probables, on peut affirmer que ces deux traits culturels, si profondément ancrés dans la structure socio-politique et rituelle des Mayas du Petén, sont non seulement pratiquement absents de notre zone d'étude, mais aussi que cette absence revêt un caractère délibéré. C'est donc sur la nature même de l'organisation sociale régionale que l'on doit se pencher pour tenter d'expliquer ce rejet.

\section{UNE AUTRE FORME DE POUVOIR}

Dans la région de Río Bec, la dispersion de groupes de dimensions comparables et l'absence apparente de grandes concentrations suggèrent un éclatement entre une multitude d'entités locales équivalentes. Pour une raison qui nous échappe encore, la région demeure à l'écart des grands courants qui ont caractérisé l'histoire de la dynastie Kaan (Arnauld et al. 2013 ; Nondédéo et al. 2010), qu'il s'agisse de Dzibanché ou de Calakmul. Inversement, la dynamique régionale aurait vu une forte compétition entre des familles désireuses d'asseoir leur autorité (Arnauld et Lacadena 2004) au travers de la construction d'édifices monumentaux, sans parvenir à s'imposer en tant que véritables dynasties (Arnauld et al. 2013). Leur statut social inférieur aux élites d'autres cités, comme le caractère éphémère de leur puissance, les aurait privées de la capacité de revendiquer l'ensemble des prérogatives et des symboles du pouvoir. Simultanément, la majorité des sites et groupes Río Bec compte au moins un, voire plusieurs édifices qui arborent des insignes de pouvoir, comme les tours, souvent décorées de masques, des représentations du monstre terrestre ou des portes zoomorphes (Nondédéo et Patrois 2007). Dans de rares cas, on peut ajouter à cette courte liste, d'ordre archéologique, les stèles ou les terrains de jeu de balle (Groupes II et V, Kajtún). Des symboles de pouvoir se rencontrent donc dans de nombreux groupes sous des formes distinctes et le plus souvent dissociées. De plus, ces symboles caractérisent aussi bien des sites majeurs comme Becán que des groupes secondaires, La Tortuga, Ramonal B (Nondédéo et Patrois 2007) ou le Groupe B de Rio Bec (Arnauld et Lacadena 2004).

Pour ce qui est des quelques stèles, à l'exception de la stèle 1 de Pasión del Cristo (Proskouriakoff 1950, figure 75a), elles montrent toutes un personnage central richement vêtu, de face, le visage de profil, ou en position assise. Les inscriptions associées, quand elles sont lisibles, donnent le nom du personnage, des dates et Lacadena a identifié au moins un glyphe-emblème à Kajtún, sur la stèle 1 (Lacadena 2004b; Nondédéo et Lacadena 2004 ; Lacadena 2007). Il a enregistré la présence d'une structure comparable de type glyphe-emblème signifiant peut-être le même titre royal (B'olonil ajaw, seigneur de B'olonil) sur trois monuments distincts, de Kajtún, déjà cité, et des Groupes II et V. Sur la stèle 3 de 
Becán, certains déchiffrements permettent d'identifier un toponyme et peut-être deux titres, ajaw et kalomté (Mayer 2009). Ces observations confirment que les rares stèles de la région de Rio Bec sont similaires, dans leur iconographie et leur contenu textuel, à celles des Basses Terres mayas centrales. Par analogie, on peut donc envisager que les personnages représentés sont les dirigeants des sites et groupes respectifs. L'existence d'un glyphe-emblème renforcerait cette proposition. Mais, d'une part, les stèles des Groupes II et Dzibil/Kajtún sont érigées dans des groupes quasi vides ou abandonnés, à cette époque. D'autre part, le placement chronologique différent de ces trois groupes soulève la question de la continuité de ce pouvoir (Michelet et al. 2008). Les personnages représentés sur les stèles représentent-ils un dirigeant précis, dans le sens des figurations des rois mayas des Basses Terres centrales, ou, à l'inverse, s'agit-il de divers personnages, chefs de lignages ou dirigeants locaux, associés à une entité géopolitique définie ?

Tout cela renvoie en définitive à la pénurie des sites qui comptent des stèles. Si nous envisageons la répartition des stèles en fonction de la localisation des autres manifestations de pouvoir (édifices à tours, portes zoomorphes, représentations du monstre terrestre), on observe qu'à l'exception de Becán, du Groupe V de Río Bec et peut-être de Pechal, nous sommes plutôt confrontés à une logique d'exclusion mutuelle. La majorité des groupes de Río Bec ne comportent pas de stèles (ni de terrains), ce qui ne les prive aucunement d'utiliser d'autres symboles ou d'autres expressions du pouvoir. Chaque entité semble exprimer sa puissance au travers d'un ensemble propre de signes, en puisant dans un fonds commun maya pour en extraire quelques éléments choisis, sans maintenir la cohérence globale. Dans ces conditions, le pouvoir serait incarné dans un groupe social, implanté sur un lieu précis. L'édifice principal du groupe, base de la Maison, en constituerait le siège. Cela expliquerait la dispersion des symboles tout comme leur diversité locale. Les stèles et les terrains ne seraient alors qu'une expression spécifique, particulière à un lieu, de la puissance locale du lignage sur son domaine.

L'absence de centres politiques, à l'exception de Becán, la répartition presque continue des groupes, constatée aux alentours de Becán (Thomas 1981) comme à la périphérie de Río Bec (Nondédéo 2003 ; Michelet et al. 2004, 2005b, 2006, 2007, 2008), l'organisation en groupes peu différenciés et de dimensions comparables, la préférence accordée à des formes architecturales originales (tours, résidences) et à une iconographie du pouvoir reposant sur des symboles choisis (masques, stèles, décors géométriques) sont autant d'indices d'une organisation socio-politique qui diverge de celle de la plupart des cités mayas du Petén (Bueno 1999). La préférence accordée aux représentations du monstre terrestre traduirait une volonté de se focaliser sur la possession de la terre productrice (Patrois 2013). À la différence d'autres entités politiques (Yaxchilan, Piedras Negras) où la hiérarchisation des cités semble traduire une organisation socio-politique pyramidale (Golden et Scherer 2013), avec un centre principal, où réside la dynastie dirigeante, dont le pouvoir est relayé par des 
centres secondaires contrôlés par des seigneurs (batabs, sajals), aucun site Río Bec, Becán inclus, ne semble exercer cette prééminence, ni disposer de la totalité des symboles ou des responsabilités du pouvoir dynastique. Bien plus, les liens de confiance ou de fidélité, tissés dans d'autres entités entre les élites locales et le pouvoir dynastique, semblent ici laisser place à une émulation compétitive.

Les études en cours permettent de dégager les informations indispensables pour définir cette structure sociale dont on commence à connaître les grandes lignes. Leur concordance suggère que nous sommes confrontés à une strate sociale de petits nobles, enracinés dans un monde essentiellement agraire, qui, sans ignorer les symboles du pouvoir dynastique, préfèrent mettre l'accent sur l'aspect architectural, la résidence et les symboles associés, délaissant d'autres aspects comme la sculpture ou les terrains. Le concept de société à Maisons, dont l'adéquation aux données du projet a été examinée campagne après campagne, correspond parfaitement à cette situation (Arnauld et al. 2013). En l'état actuel des connaissances, on interprète les données comme représentatives de la faiblesse d'un pouvoir centralisé de type dynastique, tel qu'il est documenté pour la majeure partie des sites du Petén, au profit donc de groupes nobiliaires concurrents, cherchant à affirmer leur identité et leur ancrage rural (Fowler et Arnauld 2013 ; Nondédéo et al. 2013). S’il est encore difficile d'établir les relations entre ces groupes, la faiblesse même d'un pouvoir dynastique réduirait d'autant le recours nécessaire aux symboles traditionnels de ce pouvoir. La rareté de traits aussi significatifs que les stèles, les inscriptions ou les terrains de jeu de balle trouve ainsi sa place dans une conception holistique de la société qui a donné naissance au style Río Bec. Cette conception est fondée principalement sur un rejet délibéré d'un pouvoir royal trop puissant s'exerçant au cœur d'un centre doté d'une architecture publique, au siège d'une cour royale attirant et fixant les populations. La faiblesse des rois et la dispersion des maisons nobles (Nobles Farmers and Weak Kings, Ancient Mesoamerica, 24-2, Special section 2013), gages de la société Río Bec, justifient la rareté des terrains de jeux de balle et des monuments à inscriptions.

* Manuscrit reçu en mars 2014, accepté pour publication en octobre 2014.

\section{Notes}

1. Selon Nondédéo (com. pers. 2010), une $45^{\mathrm{e}}$ stèle aurait été identifiée à Peor es Nada.

2. L'existence d'un terrain à Naachtun a été depuis confirmée, et il a fait l'objet de fouilles : sa première étape de construction remonte au Classique ancien (Michelet, com. pers. 2013).

3. Une estimation globale de l'auteur (Taladoire 2010) enregistre 350 terrains pour l'ensemble des Basses Terres mayas. Pour les Basses Terres centrales et méridionales, le total s'élève à 215 exemples, contre 135 dans les Basses Terres septentrionales. Mais le nombre de nouvelles découvertes s'accroît plus rapidement pour ces dernières.

4. Pour la région Chenes, un seul terrain confirmé existe à Santa Rosa Xtampak. Un deuxième exemple vient d'être identifié dans cette région, notoirement sous-explorée, sur le site de San Fernando. 
5. Pour illustrer ce point, il suffit de renvoyer à la récente découverte de Chactún, qui compte deux terrains et 19 monuments sculptés. Špracj (2013) souligne par ailleurs que cette région de près de $3000 \mathrm{~km}^{2}$ souffre d'un manque de recherches.

6. Depuis la rédaction de cet article, un nouveau terrain a été identifié sur le site de Lagunita, qui compte par ailleurs divers monuments Le terrain est apparemment simple, ouvert, et assez semblable aux terrains de Río Bec (Špracj 2014).

7. Un terrain de Dzibanché serait actuellement en cours de fouille.

8. De façon générale, les terrains tendent à se localiser dans les parties basses des sites, comme le montrent les exemples de Copán, d'Uxmal, de Toniná ou de Nakum pour ne citer que certains des plus connus.

9. Si l'on accepte ce placement chronologique, les deux terrains de Río Bec seraient antérieurs à celui de Becán.

10. "The ballcourt at Becán, Str. XI is very definitely a ballcourt. In 1979 during the first year of the Tulane University project at Becán directed by E. Wyllys Andrews IV, the excavation of the ballcourt was initiated. They found the remains of the center marker. Andrews found several carved pieces of the marker on the surface as the structure was being cleared in preparation for the excavations. Subsequently, a cache was found under the center markers' original position. It was a Paaktzatz Modeled lidded effigy that is illustrated in Ball's dissertation, Figure 38-C. Clearly, the ballcourt is a Late Classic structure. ”

11. L'absence de terrains n'implique d'ailleurs pas l'ignorance du jeu : comme l'a remarqué Baudez (2002), l'un des personnages des peintures murales de Santa Rita porte en sautoir un bijou qui représente une maquette de terrain, comparable à celle de la tombe 7 de Monte Alban. Il peut certes s'agir d'un objet hérité, mais, même si telétait le cas, le fait de le porter sur soi implique que son sens ne s'est pas totalement perdu. Une représentation en coupe de terrain figure d'ailleurs dans le codex de Dresde.

\section{RÉFÉRENCES CITÉES}

Adams Richard E. W.

1999 Río Azul. An ancient Maya city, University of Oklahoma Press, Norman.

Adams Richard E. W. (comp.)

1975 Preliminary reports on archaeological investigations in the Río Bec area, Campeche, Mexico, Middle American Research Institute, Tulane University (Publication, 31), New Orleans, [1974], pp. 103-146.

AndRews George F.

1999 Pyramids and palaces, monsters and masks. Volume III. Architecture of the Río Bec region and miscellaneous subjects, Labyrinthos, Culver City.

Arnauld Marie-Charlotte, Véronique Breuil-Martínez, Erick Ponciano Alvarado (éd.) 2004 La Joyanca (La Libertad, Guatemala), antigua ciudad maya del noroeste del Petén, CEMCA/Asociasión Tikal/CIRMA, Guatemala.

Arnauld Marie-Charlotte, Alfonso Lacadena

2004 «Asentar su autoridad: banquetas en el grupo B de Río Bec (Campeche, México) », Journal de la Société des américanistes, 90 (1), pp. 203-222.

Arnauld Marie-Charlotte, Dominique Michelet, Philippe NondéDÉo

2013 « Living together: co-residence, rank and alliance », Ancient Mesoamerica, 24 (2), pp. 469-493. 
BALL Joseph W.

1977 The archaeological ceramics of Becán, Campeche, Mexico, Middle American Research Institute, Tulane University (Publication, 43), New Orleans.

BAUDEZ Claude-François

1984 «Le roi, la balle et le maïs. Images du jeu de balle maya », Journal de la Société des américanistes, 70, pp. 139-152.

2002 Une histoire de la religion des Mayas, Albin Michel (Bibliothèque Histoire), Paris.

BeCQUelin Pierre, Éric Taladoire (éd.)

1990 Tonina, une cité maya du Chiapas (Mexique), CEMCA (Études mésoaméricaines, 6-4), Mexico.

Beliaev Dmitri, Alexandre Safronov

2002 Kanal kings in Quintana Roo, manuscrit.

Benavides C. Antonio

1996 «El proyecto Manzana en el sur de Campeche », in Los investigadores de la cultura maya IV, Universidad Autónoma de Campeche, Campeche, pp. 147-168.

1997 «Okolhuitz, Campeche, 1995 field season », Mexicon, XIX (2), pp. 33-35.

Bоот Eric

1991 "The Maya ballgame, as referred to in hieroglyphic writing », in G. W. van Bussel, P. L. F. van Dongen, T. J. J. Leyenaar (éd.), The Mesoamerican ballgame, Rijksmuseum voor Volkenkunde, Leyde, pp. 233-244.

BUENo C. Ricardo

1999 Entre un río de robles, un acercamiento a la arqueología de la región Río Bec, INAH (Colección científica, 411), Mexico.

2001 "Arqueología de la región Río Bec, Xpujil, Campeche », in La pintura mural prehispánica en México, Area maya, Instituto de investigaciones estéticas, UNAM, Mexico, vol. II, t. II, pp. 43-52.

BULLARD William R. Jr.

1960 "Maya settlement pattern in Northeastern Peten, Guatemala », American Antiquity, 25, pp. 355-372.

CAMPAÑa Valenzuela Luz Evelia

2005 "Contribuciones a la historia de Becán », Arqueología mexicana, 13 (75), pp. 48-56.

CARRASCO Ramón

1987 «Nuevas tapas de bóveda decoradas, en la región central de Yucatan », Mexicon, IX (1), pp. 16-20.

1994 Chicanná, Campeche, un sitio de la frontera sur. Estudio arquitectónico, Centro de estudios mayas, UNAM, Mexico.

1996 «Calakmul, Campeche », Arqueología mexicana, 3 (18), pp. 46-51. 
CARrasco Ramón, Sylviane Boucher

1987 «Las escaleras jeroglíficas de El Resbalón, Quintana Roo », in Primer simposio internacional sobre epigrafía maya, Instituto de antropología e historia de Guatemala, Guatemala, pp. 1-21.

Con Uribe María José

2000 «El juego de pelota en Cobá, Quintana Roo », Arqueología, segunda época, 23, pp. 27-50.

CORTES DE BRASDEFER Fernando

1997 «Las esculturas estucadas de Chakanbakan », Arqueología, segunda época, 18, pp. 51-60.

1998 Kohunlich, ciudad del sol, Editora Norte-Sur, Mexico.

Dzul Sara, Éric TALADOIRE

2010 «Espacio y cronología en la periferia de Río Bec», in E. Vargas, A. Benavides (éd.), La península de Yucatán: investigaciones recientes y cronologías alternativas, Universidad Autónoma de Campeche, Campeche, pp. 121-134.

EATON Jack D.

1972 «A report on excavations at Chicanná, Campeche, México », Cerámica de cultura maya, 8, pp. 42-61.

EsParza Octavio Quetzalcoatl

2006 «La Estela 2 de Pol Box: estudio de un monumento del sur de Quintana Roo », in J. P. Laporte, B. Arroyo, H. Mejía (éd.), XIX Simposio de investigaciones arqueológicas en Guatemala, 2005, Museo nacional de arqueología y etnología, Guatemala, pp. 661-669.

Esparza Olguín Octavio Q., Vania E. Pérez Gutiérrez

2009 "Archaeological and epigraphic studies in Pol Box, Quintana Roo », The PARI journal. A quarterly publication of the Pre-Columbian Art Research Institute, 9 (3), pp. 1-16.

Folan William, Raymundo González H., Ma del Rosario Domínguez C., Abel Morales L., Ivan Šprajc, Hubert R. Robichaux, Candice Pruett, Joel D. GunN

2005 «Las ruinas de Oxpemul, Campeche Mexico: una corte real fortificada en la frontera norte entre el estado regional de Calakmul y el Río Bec », in Los investigadores de la cultura maya XIII, Universidad autónoma de Campeche, Campeche, t. II, pp. 475-486.

Flores C. Alberto G., Jorge Pablo Huerta R., Dave Johnstone, Adam Kaeding, Johan Normark, Justine M. SHAw, Tatiana Young

2008 Final Report of the Cochuah Regional Archaeological Survey's 2008 Field Season, J. M. Shaw (éd.), College of the Redwoods, Eureka (CA).

Fowler William R., Marie-Charlotte ARnAuld

2013 «Special section. Introduction. Noble farmers and weak kings in the Classic Maya Lowlands: the Río Bec archaeological project, 2002-2010 », Ancient Mesoamerica, 24 (2), pp. 343-351. 
García CoOK Angel, Beatriz Leonor Merino CARríon

1998 «Cantona: urbe prehispánica en el Altiplano central de México », Latin American Antiquity, 9 (3), pp. 191-216.

GARcía Moll Roberto

1977 «Los escalones labrados del edificio 33, Yaxchilan, Chiapas », Revista mexicana de estudios antropológicos, 23 (3), pp. 395-423.

GENDROP Paul

1983 Los estilos Río Bec, Chenes y Punc en la arquitectura maya, UNAM, Mexico.

Golden Charles, Andrew K. SCHERER

2013 « Territory, trust, growth and collapse in Classic Period Maya kingdoms », Current Anthropology, 54 (4), pp. 397-435.

Greene Robertson Merle, Edward B. KurJack, Ruben Maldonado

1991 "Ballcourts of the northern Maya Lowlands", in V. L. Scarborough, D. R. Wilcox (éd.), International symposium on the Mesoamerican Ballgame, University of Arizona Press, Tucson, pp. 249-276.

Harrison Peter D.

2005 «Some ancient Maya monuments from Southern Quintana Roo. Defining the "Triad-Pill" monument type ", in J. M. Shaw, J. P. Mathews, Quintana Roo archaeology, University of Arizona Press, Tucson, pp. 214-227.

LACADENa Alfonso

2004a "The glyphic corpus from Ek’ Balam, Yucatán », FAMSI, www.famsi.org/ reports/01057/

2004b «Operación VIIB: estudios epigráficos », in D. Michelet et al., Río Bec (Campeche, México). Informe de la segunda temporada del 27 de enero al 25 de mayo de 2003, Consejo de arqueología, INAH, Mexico, pp. 1-11.

2006 "Operación IVB: epigrafía », in D. Michelet et al., Río Bec (Campeche, México). Informe de la cuarta temporada del 6 de febrero al 8 de mayo de 2005, Consejo de Arqueología, INAH, Mexico, pp. 9-17.

2007 «Las inscripciones de Río Bec », communication présentée au symposium «Río Bec: génesis, desarrollo y percepción de un fenómeno cultural», P. Nondédéo (coord.), VIIo Congreso internacional de los mayistas, 8-14 de julio, Mérida.

López Camacho Javier, Kenichiro Tsukamoto

2003 «Levantamiento topográfico de El Resbalón, Quintana Roo, México », in J. P. Laporte, B. Arroyo, H. Mejía (éd.), XIX Simposio de investigaciones arqueológicas en Guatemala, 2002, Museo nacional de arqueología y etnología, Guatemala.

Martin Simon, Nikolaï GrubE

$2000 \quad$ Chronicle of the Maya kings and queens. Deciphering the dynasties of the Ancient Maya, Thames and Hudson, Londres.

Mathews Peter

1983 "A painted capstone at Becan, Campeche », Mexicon, 5 (4), pp. 70-71. 
MAYER Karl Herbert

1982 «Eine Maya-Inschrift aus Tzibanché, Quintana Roo, Mexiko », Mexicon, 4 (4), pp. 64-65.

1988 «A painted maya text at Chicanná, Campeche, México », Mexicon, 10 (5), pp. 87-88.

2003 "The maya ruins of San Lorenzo, Campeche, relocated », Mexicon, 25 (5), pp. 114-117.

2009 "The inscribed stela 3 from Becan, Campeche, Mexico », Mexicon, 31 (5), pp. 104-107.

Michelet Dominique, Marie-Charlotte Arnauld, Philippe NondéDÉo, Grégory Pereira, Fabienne de Pierrebourg, Éric Taladoire

1998 «La saison de fouille de 1998 à Balamku (Campeche, Méxique) : des avancées substantielles », Journal de la Société des américanistes, 84 (1), pp. 183-199.

Michelet Dominique, Éric Taladoire, Sara Dzul, Philippe Nondédéo, Julie Patrois, Charlotte Arnauld, Angeles Cantero, Pierre Becquelin, Rosarío Acosta, Emyly González, Diana Arano, Alfonso Lacadena, Didier Galop, Boris VANNIÈRE

2004 Proyecto Río Bec (Campeche Mexique). Informe de la segunda temporada, del 27 de febrero al 25 de mayo de 2003, Archivo del Consejo de arqueología, INAH, Mexico.

Michelet Dominique, Éric Taladoire, Sara Dzul, Philippe Nondédéo, Julie Patrois, Georges Marchand, Marie-Charlotte Arnauld, Laure Déodat, Astrid Huser, Pierre Becquelin, Grégory Pereira, Jorge Ortega, Emyly González, Ma. Rosalía Carrillo, Diana Arano, Didier Galop, Boris VANNIÈRE, Chloé ANDRIEU

2005a Proyecto Río Bec (Campeche, Mexique). Informe de la tercera temporada, del 16 de febrero al 15 de mayo de 2004, Archivo del Consejo de arqueología, INAH, Mexico.

Michelet Dominique, Philippe Nondédéo, Marie-Charlotte Arnauld

2005b 《Río Bec, ¿una excepción? », Arqueología mexicana, 13 (75), pp. 58-63.

Michelet Dominique, Éric Taladoire, Sara Dzul, Philippe Nondédéo, Julie Patrois, Marisa Vásquez, Alfonso Lacadena, Georges Marchand, Charlotte Arnauld, Laure Déodat, Sophie Lardé, Céline Gillot, Grégory Pereira, Amparo Robles, Emyly González, Ma. Rosalía Carrillo, Diana Arano, Didier Galop, Boris Vannière, Christine Heinz, Nicolas Latsanopoulos

2006 Proyecto Río Bec (Campeche, Mexique). Informe de la cuarta temporada, del 8 de febrero al 6 de mayo de 2005, Archivo del Consejo de arqueología, INAH, Mexico.

Michelet Dominique, Charlotte Arnauld, Philippe Nondédéo, Éric Taladoire, Sara Dzul, Chloé Andrieu, Laure Déodat, Céline Gillot, Julie Patrois, Grégory Pereira, J. Joel Hernández O., Georges Marchand, Agnès 
Stock, Boris Vannière, Éva Lemonnier, Christine Heinz, Emyly GonzÁlez, Ma. Rosalía CARrillo, Yareli Jaídar, Nicolas Latsanopoulos 2007 Proyecto Río Bec (Campeche, Mexique). Informe de la quinta temporada, del 5 de febrero al 4 de mayo de 2006, Archivo del Consejo de arqueología, INAH, Mexico.

Michelet Dominique, Charlotte Arnauld, Philippe Nondédéo, Éric Taladoire, Sara Dzul, Chloé Andrieu, Laure Déodat, Céline Gillot, Julie Patrois, Alfonso Lacadena, Ignacio Cases, Grégory Pereira, José D. Álvarez, Gabriel Francia G., Éva Lemonnier, Emyly González, Ma. Rosalía CARrillo, Yareli Jaídar, Adela VÁsquez, Nicolas Latsanopoulos

2008 Proyecto Río Bec (Campeche, Mexique). Informe de la sexta temporada, del 4 de febrero al 3 de mayo de 2007, Archivo del Consejo de arqueología, INAH, Mexico.

NALDA Enrique, Luz Evelia CAMPAÑa, Javier LóPEz C.

1994 «Sur de Quintana Roo: Dzibanché y Kinichná », Arqueologia mexicana, 2 (10), pp. 14-19.

NonDÉDÉO Philippe

2003 L'Évolution des sites mayas du Sud de l'État du Campeche, Mexique, Archaeopress (BAR. International series, 1171; Paris monographs in American archaeology, 12), Oxford.

NondéDÉo Philippe, Alfonso LaCADEna

2004 "Kajtún: un nuevo sitio con monumentos esculpidos en la región Río Bec ", Journal de la Société des américanistes, 90 (1), pp. 183-201.

2007 New data, new readings from Pechal, Campeche, manuscrit, archives du projet Río Bec, Nanterre.

NondÉDÉo Philippe, Julie PATroIS

2007 «Iconografía del poder en la región Río Bec: representaciones y primeras interpretaciones », in E. Vargas, A. Benavides (éd.), El patrimonio arqueológico maya en Campeche. Novedades, afectaciones, soluciones, UNAM (Cuadernos del Centro de estudios mayas, 35), Mexico, pp. 159-205.

NondÉDÉo Philippe, Marie-Charlotte Arnauld, Dominique Michelet

2013 «Rio Bec Settlement patterns and local socio-political organization », Ancient Mesoamerica, 24 (2), pp. 373-396.

NondédÉo Philippe, Julie Patrois, Alfonso Lacadena, Marie-Charlotte Arnauld, Éric TAladoire, Dominique Michelet

2010 «De la autonomía política y cultural de la provincia de Río Bec », Estudios de cultura maya, 36, pp. 37-66.

NORMARK Johan

2009 «Ballcourts in the Cochuah region », https://haecceities.wordpress.com/

Patrois Julie

2013 «Río Bec graffiti: a private form of art», Ancient Mesoamerica, 24 (2), pp. $433-447$ 
PESCADOR Laura

1998 «Nadzca'an », Journal de la Société des américanistes, 84 (1), pp. 167-182.

ProskouriakofF Tatiana

1950 A study of Classic Maya sculpture, Carnegie Institution (Publication, 593), Washington.

RAESFELD Lydia

1990 «New discoveries at El Tajín, Veracruz », Mexicon, 12 (5), pp. 92-95.

1992 «Die Ballspielplätze in El Tajin, Mexico », Ethnologische Studien, 8.

Reese-Taylor Kathryn, Peter Mathews, Marcelo Zamora M., Debra Walker, Martin Rangel, Silvia Alvarado, Ernesto Arredondo, Shawn Morton, Alex Parmington, Roberta Parry, Baudilio Salazar, Jeff SeIbert

2005 "Proyecto arqueológico Naachtun: resultados preliminares de la primera temporada de campo 2004 », in J. P. Laporte, B. Arroyo, H. E. Mejía (éd.), XVIII Simposio de investigaciones arqueológicas en Guatemala, 2004, Ministerio de Cultura y Deportes/Instituto de antropología e historia/ Asociación Tikal/Foundation for the advancement of Mesoamerican studies Inc., Guatemala, pp. 91-100.

RUPPERT Karl, John H. DENISON Jr.

1943 Archaeological reconnaissance in Campeche, Quintana Roo and Peten, Carnegie Institution (Publication, 543), Washington.

RuZ LHUILLIER Alberto

1945 "Campeche en la arqueología maya », Acta Antropológica, 1 (2-3).

Shaw Justine M., Jennifer P. Mathews

2005 Quintana Roo archaeology, The University of Arizona Press, Tucson.

ŠPRACJ Ivan

2013 "Chactún, un sitio maya en el sureste de Campeche », Mexicon, 35 (4), pp. 81-82.

2014 «Lagunita and Tamchén, two major sites in southeastern Campeche », Mexicon, 36 (5), pp. 134-136.

Špracj Ivan, Atasta Flores E., Daniel Juárez C., Adrián BaKer P., María Isabel García L., Nikolaï Grube, Tomaž PodobNiKar, Krištof Ostir

2008 Reconocimiento arqueológico en el Sureste de Campeche, México: 1996-2005, Archaeopress (BAR. International series, 1742; Paris monographs in American archaeology, 19), Oxford.

2010 «Archaeological reconnaissance in Southeastern Campeche, Mexico: summary of the 2007 field season », Mexicon, 32 (6), pp. 148-154.

ŠPracu Ivan, Atasta Flores EsQuivel

2010 «El Zacatal », Mexicon, 32 (1), pp. 2-5.

ŠPracj Ivan, Florentino García Cruz, Héber Ojeda Mas

1997 "Reconocimiento arqueológico en el sureste de Campeche », Arqueología, segunda época, 18, pp. 29-49. 
TALADOIRE Éric

1980 «Réévaluation d'un des critères de définition des centres cérémoniels majeurs dans les Basses Terres mayas ", Boletín (MAEFM), 2, pp. 39-53.

1981 Les Terrains de jeu de balle en Mésoamérique et dans le Sud-Ouest des États-Unis, MAEFM (Études Mésoaméricaines, 2 [4]), Mexico.

1993 «Los juegos de pelota en el norte de Yucatan: una revisión de los datos », in Ma. J. Iglesias Ponce de Leon, F. Ligorred Perramon (éd.), Perspectivas antropológicas en el mundo maya, Sociedad española de estudios mayas, Madrid, pp. 163-180.

Taladoire Éric, Sara Dzul, Philippe NondÉdÉo, Mélanie Forné

2013 "Chronology of occupation at Río Bec: sequences and datation », Ancient Mesoamerica, 24 (2), pp. 353-372.

Thomas Prentice M. Jr.

1981 Prehistoric Maya settlement patterns at Becán, Campeche, Mexico, Middle American Research Institute, Tulane University (Publication, 45), New Orleans.

Thompson John E. S.

1953 "A stela at San Lorenzo, southeastern Campeche", Notes on Middle American Achaeology and Ethnology, 115, pp. 228-231.

VARGas P. Ernesto, Angélica Delgado S.

2003 "El Clásico terminal en El Tigre, Campeche», in Los investigadores de la cultura maya XI, Universidad autónoma de Campeche, Campeche, t. II, pp. 406-423.

Wren Linnea, Travis NygARD

2005 "Witnessed at Yo'okop: images and texts of rulers in a watery realm », in J. M. Shaw, J. P. Mathews, Quintana Roo Archaeology, University of Arizona Press, Tucson, pp. 166-183. 
